\title{
Neutrons et matériaux : introduction
}

\section{J. Schweizer}

CEA-Grenoble, DRFMC/SPSMS/Magnétisme et Diffraction Neutronique, 38000 Grenoble, France

I- Généralités sur les neutrons

I-1 Les propriétés du neutron

I-2 Ondes et corpuscules, les phénomènes de base. Sections efficaces.

I-3 Les interactions neutron-matière.

I-4 Production de faisceaux de neutrons.

I-5 Détection des neutrons

II- La diffusion des neutrons : un coorréloscope dans l'espace $(Q, \omega)$

II-1 La mesure des neutrons diffusés : une mesure dans l'espace (Q,w), transformé de Fourier de l'espace $(\mathrm{r}, \mathrm{t})$.

II-2 Sections efficaces cohérentes et incohérentes

II-4 Gain et perte d'énergie. Le principe de la balance détaillée

II-5 Diffusion élastique et diffusion inélastique

II-6 Les instruments de diffusion neutronique

III- Ce que l'on peut voir avec les neutrons

III-1 Voir la structure

III-2 Voir la dynamique

III-3 Voir le couplage entre dynamique et structure

\section{Conclusion}

Bibliographie

\section{Généralités sur les neutrons}

\section{I-1 Les propriétés du neutron}

Le neutron est une particule élémentaire qui a été découverte en 1932 par James Chadwick. Ses caractéristiques principales sont résumées dans le tableau I. II a été utilisé pour la première fois par Clifford Shull1 en 1946 comme outil pour des! expériences de diffusion. Cette technique s'est depuis constamment développée pour concerner tous les aspects de la matière condensée: physique, chimie, matériaux, biologie. II s'agit d'un outil tout à fait exceptionnel car le neutron possède des propriétés uniques et particulièrement adaptées pour ces études.

\footnotetext{
1 Clifford Shull, prix Nobel 1994 avec Bertram Brockhouse est décédé le 31 mars 2001 aux Etats Unis.
} 
Tableau I: Caractéristiques du neutron, relations et constantes utiles

\begin{tabular}{|ll|}
\hline masse & $\mathrm{m}=1.00866 \mathrm{uma}$ \\
spin & $\mathrm{m}=1.67510^{-27} \mathrm{~kg}$ \\
moment magnétique & $\mathrm{s}=1 / 2$ \\
& $\mu_{\mathrm{n}}=9.66310^{-27} \mathrm{JT}-1$ \\
& $\mu_{\mathrm{n}}=1.913 \mu_{\mathrm{BN}}$ \\
décomposition & $\mathrm{n} \rightarrow \mathrm{p}+\mathrm{e}^{-}+\mathrm{v}$ \\
temps de vie & $\tau=888 \mathrm{~s}$ \\
demi-vie & $\mathrm{T}_{1 / 2}=\tau \log 2=615 \mathrm{~s}$ \\
& $\lambda=\frac{\mathrm{h}}{\mathrm{mv}}$ \\
\hline longueur d'onde & $\overrightarrow{\mathrm{k}}\left(\mathrm{k}=\frac{2 \pi}{\lambda}\right)$ \\
vecteur d'onde & $\overrightarrow{\mathrm{p}}=\hbar \overrightarrow{\mathrm{k}}$ \\
moment & $\mathrm{E}=\frac{1}{2} \mathrm{mv}^{2}=\frac{\hbar^{2} \mathrm{k}^{2}}{2 \mathrm{~m}}$ \\
énergie & $\mathrm{h}=6.62610^{-34} \mathrm{J.s}$ \\
& $\hbar=1.05410^{-34} \mathrm{J.s}$ \\
& $\mu_{\mathrm{B}}=9.27410^{-24} \mathrm{JT}-1$ \\
& $\mu_{\mathrm{BN}}=5.05110^{-27} \mathrm{JT}-1$ \\
& $1 \mathrm{eV}=1.60210^{-19} \mathrm{~J}$ \\
\hline
\end{tabular}

I. 1. 1. Le neutron est électriquement neutre.

Le neutron possède une charge électrique nulle. De ce fait, il peut pénétrer la matière sans se faire arrêter par une barrière coulombienne. Contrairement aux rayons $X$ qui ne voient, la plupart du temps, que la surface de la matière, les neutrons sondent l'intérieur des échantillons, et c'est là un atout important.

I. 1. 2. Le neutron est une particule, mais en même temps une onde, avec une longueur d'onde associée donnée par la formule de de Broglie:

$$
\lambda=\frac{\mathrm{h}}{\mathrm{mv}}
$$

où $\mathrm{h}$ est la constante de Planck, $\mathrm{m}$ la masse du neutron et $\mathrm{v}$ sa vitesse.

Avec des neutrons thermalisés par un modérateur dont la température est $300 \mathrm{~K}$, leur vitesse moyenne est de $2200 \mathrm{~m} / \mathrm{s}$ et leur longueur d'onde moyenne de $1.8 \AA ̊ A$, c'est à dire une longueur d'onde qui est du même ordre de grandeur que les distances inter atomiques dans la matière condensée. Les neutrons thermiques sont donc tout à fait adaptés pour être diffractés par les atomes de la matière qui nous entoure.

Pour les photons, rappelons le, la longueur d'onde est donnée par :

$$
\lambda=\frac{c}{v}
$$


où $\mathrm{c}$ est la vitesse de la lumière et $v$ la fréquence de la radiation. C'est ainsi que ce sont les rayons $X$, avec des fréquences de $10^{17}$ à $10^{19} \mathrm{~Hz}$ qui correspondent à des longueurs d'onde comparables aux distances entre atomes et molécules.

I. 1. 3. L'énergie du neutron correspond à l'énergie cinétique de la particule et est donnée par la formule classique:

$$
\mathrm{E}=\frac{1}{2} \mathrm{mv} v^{2}=\frac{\hbar^{2} \mathrm{k}^{2}}{2 \mathrm{~m}}
$$

où $k$ est le nombre d'onde $(k=2 \pi / \lambda)$.

Cette énergie se retrouve en joules si on exprime la masse en kilogrammes et la vitesse en $\mathrm{m} / \mathrm{s}$. En pratique, on utilise l'électron volt comme unité d'énergie.

Compte tenu de la formule (1) qui relie la longueur d'onde et la vitesse, on arrive à :

$$
\mathrm{E}=\frac{\mathrm{h}^{2}}{2 \mathrm{~m} \lambda^{2}}
$$

ou, à la formule équivalente, avec des unités bien spécifiées:

$$
\lambda(\text { en } \AA) \cdot \sqrt{\mathrm{E}}(\mathrm{E} \text { en eV) }=0.286
$$

Ainsi, les neutrons thermiques (longueurs d'onde entre 1 et $5 \AA$ ) ont des énergies dans la gamme des $\mathrm{meV}$, c'est à dire, dans la gamme des excitations de la matière condensée.

Rappelons que pour les photons l'énergie est donnée par :

$$
E=h . v
$$

et que pour des rayons $X$ de longueur d'onde $1 \AA$, $E$ vaut $12,4 \mathrm{keV}$, soit 6 ordres de grandeur de plus que les excitations dans la matière condensée. Pour faire de la spectroscopie de la matière condensée, il faut utiliser le visible ou l'infrarouge.

Le neutron est la seule sonde qui permet d'avoir accès en même temps à la structure, par des phénomènes d'interférence, et à la dynamique, par spectroscopie.

\section{1. 4. Le neutron porte un spin $s=1 / 2$.}

II lui est donc associé une fonction d'onde de spin qui agit dans un espace à deux dimensions sous tendu par les deux vecteurs $\mid+>$ et $\mid->$ :

$$
\chi=a|+>+b|->
$$

Il en résulte deux conséquences principales :

D'une part il est possible de polariser les faisceaux de neutrons et aussi d'analyser la polarisation des neutrons après qu'ils aient interagi avec l'échantillon. Ceci donne, comme pour les rayons $X$, toute une gamme de possibilités expérimentales.

D'autre part, le neutron porte un moment magnétique associé au spin. Ce moment est petit et vaut $1.91 \mu_{\mathrm{BN}}$ (magnétons de Bohr nucléaires). Mais ce moment interagit avec tous les champs magnétiques et, en particulier, ceux qui sont créés par les moments magnétiques d'origine électronique et qui sont mille fois plus grands que 
lui. Cette interaction donne accès aux structures magnétiques et permet l'étude des excitations magnétiques.

\section{I-2 Ondes et corpuscules, les phénomènes de base. Sections efficaces}

Comment se comporte un faisceau de neutrons dans le vide et dans la matière? Nous allons passer en revue dans ce paragraphe les phénomènes que l'on rencontre.

\section{2. 1 Faisceau monochromatique}

Un faisceau monochromatique de neutrons peut être considéré comme une onde plane. On peut écrire une onde qui se propage selon la direction $\bar{z}$ par la formule:

$$
\Psi=\mathrm{e}^{i(\mathrm{kz}-2 \pi v \mathrm{t})}
$$

où $v$ est la fréquence de l'onde plane. La photo instantanée d'une telle onde est représentée sur la figure 1a. Les lignes de crête sont figurées par les traits pointillés perpendiculaires à la direction de propagation. Ces lignes de crête sont séparées par une longueur d'onde $\lambda$. Le vecteur d'onde $\overrightarrow{\mathbf{k}}$ est un vecteur parallèle à la propagation et de longueur $k=2 \pi / \lambda$ et le moment des neutrons est

$$
\overrightarrow{\mathrm{p}}=\mathrm{m} \overrightarrow{\mathrm{v}}=\hbar \overrightarrow{\mathrm{k}}
$$

En tout point de l'espace l'onde est périodique dans le temps, avec une pulsation $\omega$ et une période T données par:

$$
\omega=2 \pi / T=2 \pi . v
$$

a) Faisceau monochromatique

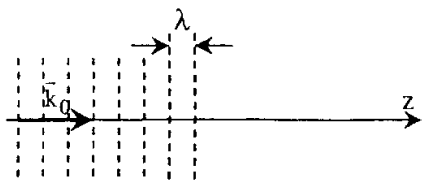

b) Absorption

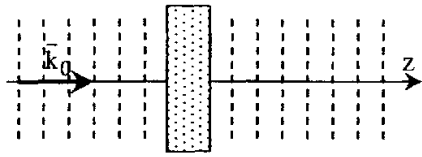

c) Diffusion

Figure 1: Les phénomènes de base

d) Réfraction
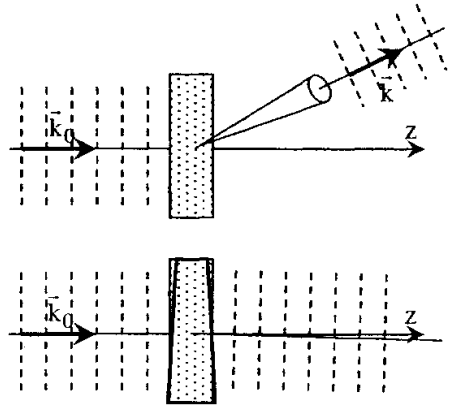


\section{2. 2 Absorption d'un faisceau de neutrons}

Lorsqu'un faisceau de neutrons traverse de la matière, une partie de ces neutrons peut être absorbée par les noyaux des atomes pour exciter ces noyaux et finalement leur faire éjecter un proton, une particule $\alpha$, un photon $\gamma$ ou même conduire à une fission (figure 1b). Si l'échantillon est une plaque mince où les atomes ne se portent pas ombre les uns sur les autres, le nombre de réactions par seconde $R$ est proportionnel à l'intensité du faisceau $I_{0}$ mesuré en neutrons par seconde et à la concentration $\mathrm{n}$ de noyaux sur la plaque (nombre de noyaux par unité de surface) :

$$
\left.R(\text { nbre réactions } / \mathrm{s})=I_{0}(\text { nbre neutrons } / \mathrm{s}) \cdot \mathrm{n} \text { (nbre noyaux } / \mathrm{cm}^{2}\right) \cdot \sigma_{\mathrm{a}}
$$

La constante de proportionnalité $\sigma_{a}$ a donc les dimensions d'une surface et s'exprime habituellement en barns $\left(1\right.$ barn $\left.=10^{-24} \mathrm{~cm}^{2}\right)$. C'est la section efficace d'absorption qui caractérise la capacité de chaque noyau à absorber les neutrons.

Si l'échantillon est maintenant une plaque plus épaisse où il faut tenir compte, pour chaque couche, de l'atténuation du faisceau due à l'absorption par les couches précédentes, l'intensité du faisceau à la sortie de la plaque est donnée par :

$$
\mathrm{I}=\mathrm{I}_{0} \mathrm{e}^{-\mu \mathrm{t}}
$$

où t est l'épaisseur de la plaque et où le coefficient d'absorption linéaire $\mu$ est donné par:

$$
\mu=N \cdot \sigma_{\mathrm{a}}
$$

$\mathrm{N}$ représentant cette fois le nombre de noyaux par unité de volume.

Alors que pour les rayons $X, \mu$ est de l'ordre de 100 à $1000 \mathrm{~cm}^{-1}(0.01$ à $0.001 \mathrm{~cm}$ de matière suffisent pour affaiblir le faisceau d'un facteur $1 / e$ ), la matière est beaucoup plus transparente pour les neutrons car $\mu$ est compris entre 0.01 et $1 \mathrm{~cm}^{-1}$ pour la plupart des matériaux.

L'absorption est un phénomène général des radiations: il dépend de l'énergie et donc de la longueur d'onde des neutrons. On peut le comparer au problème d'un oscillateur amorti et forcé. En effet, il existe des énergies de résonance pour les neutrons qui produisent des excitations du noyau composé (noyau + neutron). Au voisinage de ces énergies de résonance l'absorption est très forte et elle diminue au fur et à mesure qu'on s'en éloigne. Pour presque tous les noyaux, ces énergies de résonance se trouvent bien au delà des énergies des neutrons thermiques et la section efficace d'absorption $\sigma_{a}$ est alors proportionnelle à $1 / v$ ou à $\lambda$. C'est pourquoi les tables d'absorption comme le tableau II précisent toujours la vitesse ou la longueur d'onde pour laquelle ces sections efficaces sont données, laissant à l'expérimentateur le soin d'en déterminer la valeur pour la longueur d'onde qu'il utilise.

Mais pour certains noyaux, souvent considérés comme pathologiques ${ }^{2}$, une ou plusieurs énergies de résonance se trouvent dans la gamme d'énergie des neutrons

2 Il s'agit principalement des noyaux suivants: ${ }^{3} \mathrm{He},{ }^{6} \mathrm{Li},{ }^{10} \mathrm{~B},{ }^{113} \mathrm{Cd},{ }^{115} \mathrm{In},{ }^{123} \mathrm{Te},{ }^{149} \mathrm{Sm},{ }^{151} \mathrm{Eu},{ }^{155} \mathrm{Gd},{ }^{157} \mathrm{Gd}$, ${ }^{164} \mathrm{Dy},{ }^{168} \mathrm{Yb}$ et ${ }^{176} \mathrm{Lu}$. 
Figure 2: Section efficace totale du noyau ${ }^{103} \mathrm{Rh}$

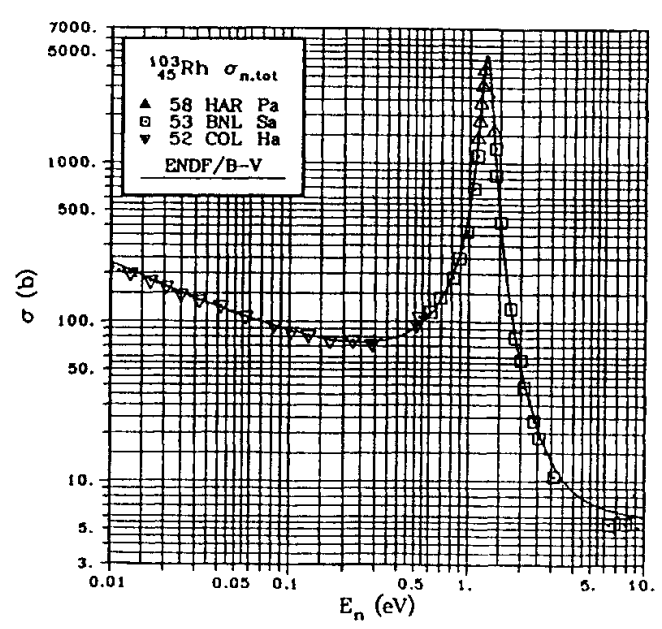

utilisés. Il en résulte que l'absorption est très grande (des centaines ou des milliers de barns) et que cette absorption varie très rapidement avec l'énergie des neutrons. Pour la connaître, des tables comme la table précédente ne suffisent plus, il faut consulter la bible: le "Neutron Cross Sections" [1] qui donne les variations de sections efficaces en fonction de l'énergie des neutrons pour tous les éléments naturels et pour tous les noyaux. A titre d'exemple, la figure 2 représente la section efficace totale (absorption + diffusion) du ${ }^{103} \mathrm{Rh}$ qui présente une résonance à $1.3 \mathrm{eV}$ et suit une loi en $1 / \mathrm{v}$ entre 10 et $100 \mathrm{meV}$.

Il faut noter que c'est avec ces éléments pathologiques, en profitant de leurs caractéristiques d'absorption tout à fait particulières pour les neutrons thermiques, que l'on réalise les différents dispositifs expérimentaux comme les détecteurs, les filtres ou la collimation des faisceaux.

\section{2. 3 Diffusion des neutrons}

La figure 1c représente le phénomène de diffusion. Suite à l'interaction entre le faisceau de neutrons incidents et la matière de l'échantillon, l'échantillon se comporte comme une source et produit une onde diffusée :

$$
\Psi_{d i f}=-\mathrm{f}\left(\overrightarrow{\mathrm{k}}, \overrightarrow{\mathrm{k}}^{\prime}\right) \frac{\mathrm{e}^{i\left(\mathrm{k}^{\prime} \mathrm{r}-2 \pi v \mathrm{t}\right)}}{\mathrm{r}}
$$

où l'amplitude de diffusion, $f\left(\vec{k}, \vec{k}^{\prime}\right)$, avec la dimension d'une longueur, caractérise la force de l'interaction neutron-matière. A grande distance de la cible, l'onde diffusée pourra être considérée comme une onde plane, de vecteur d'onde $\vec{k}^{\prime}$. Le vecteur représentant la différence entre les deux vecteurs d'onde, est appelé le vecteur de diffusion :

$$
\overrightarrow{\mathrm{Q}}=\overrightarrow{\mathrm{k}}-\overrightarrow{\mathrm{k}}^{\prime}
$$

et le transfert de moment est $\hbar \overrightarrow{\mathrm{Q}}$.

Si la cible se réduit à une seule particule fixe, cette onde est sphérique et le processus est élastique. Si la cible est plus complexe, il peut y avoir échange 
d'énergie entre les neutrons et le système diffusant avec $\left|\overrightarrow{\mathbf{k}^{\prime}}\right| \neq|\overrightarrow{\mathrm{k}}|$. Le changement d'énergie du neutron est :

$$
\hbar \omega=\mathrm{E}-\mathrm{E}^{\prime}=\frac{\hbar^{2}}{2 \mathrm{~m}}\left(\mathrm{k}^{2}-\mathrm{k}^{\prime 2}\right)
$$

\section{Par convention, $\hbar \omega$ est positif lorsque le neutron cède de l'énergie au système cible.}

Ce qui est mesuré, dans une expérience de diffusion, c'est la fraction de neutrons d'énergie incidente $E$, diffusés dans un élément d'angle solide $d \Omega$, avec une énergie comprise entre $E^{\prime}$ et $E^{\prime}+d E^{\prime}$. C'est la section efficace différentielle partielle qui s'exprime en barns par stéradian et par unité d'énergie :

$$
\frac{\mathrm{d}^{2} \sigma}{\mathrm{d} \Omega \mathrm{dE}^{\prime}}=\frac{1}{\Phi_{0}} \frac{\text { nombre de neutrons diffusés par seconde dans } \mathrm{d} \Omega \mathrm{et} \mathrm{dE^{ \prime }}}{\mathrm{d} \Omega \mathrm{dE}^{\prime}}
$$

où $\Phi_{0}$ est le flux de neutrons incidents, c'est à dire le nombre de neutrons par seconde et par unité de surface.

Si on n'analyse pas l'énergie des neutrons diffusés, mais que l'on compte tous les neutrons diffusés dans $\mathrm{d} \Omega$, on mesure la section efficace différentielle qui s'exprime en barns par stéradian :

$$
\frac{\mathrm{d} \sigma}{\mathrm{d} \Omega}=\int \mathrm{dE} \frac{\mathrm{d}^{2} \sigma}{\mathrm{d} \Omega \mathrm{dE}}=\frac{1}{\Phi_{0}} \frac{\text { nombre de neutrons diffusés par seconde dans } \mathrm{d} \Omega}{\mathrm{d} \Omega}
$$

Enfin, si on intègre les neutrons diffusés dans toutes les directions de l'espace, on arrive à la section efficace de diffusion totale qui s'exprime en barns :

$$
\sigma_{\mathrm{dif}}=\int \mathrm{d} \Omega \frac{\mathrm{d} \sigma}{\mathrm{d} \Omega}=\frac{\text { nombre de neutrons diffusés par seconde }}{\Phi_{0}}
$$

Nous verrons dans la deuxième partie de ce cours que pour un processus de diffusion où le vecteur d'onde des neutrons passe de $\overrightarrow{\mathbf{k}}$ à $\overrightarrow{\mathbf{k}}^{\prime}$ et où la cible passe d'un état $\lambda$ à un état $\lambda^{\prime}$, la section efficace différentielle partielle est donnée par l'expression :

$$
\left(\frac{\mathrm{d}^{2} \sigma}{\mathrm{d} \Omega \mathrm{dE}^{\prime}}\right)_{\substack{\overrightarrow{\mathrm{k}} \rightarrow \overrightarrow{\mathrm{k}}^{\prime} \\ \lambda \rightarrow \lambda^{\prime}}}=\frac{\mathrm{k}^{\prime}}{\mathrm{k}}\left(\frac{\mathrm{m}}{2 \pi \hbar^{2}}\right)^{2}\left|\left\langle\overrightarrow{\mathrm{k}}^{\prime} \lambda^{\prime}|\mathrm{V}(\overrightarrow{\mathrm{r}})| \overrightarrow{\mathrm{k}} \lambda\right\rangle\right|^{2} \delta\left(\hbar \omega+\mathrm{E}_{\lambda}-\mathrm{E}_{\lambda^{\prime}}\right)
$$

où $V(\vec{r})$ est le potentiel représentant l'interaction entre le neutron et les atomes de la cible.

\section{2. 4 Réfraction des neutrons}

Le phénomène de réfraction, où le faisceau incident change sa direction au passage à travers un échantillon sans subir une perte importante d'intensité, est bien connu pour la lumière visible à travers une lentille ou un prisme. II existe aussi pour les neutrons, ainsi d'ailleurs que pour les rayons $X$. Mais dans ces deux cas les angles de déflexion sont très faibles (voir figure 1d) car, contrairement au cas de la lumière, l'indice de la matière pour les neutrons thermiques (et pour les rayons $X$ ) est très 
voisin de 1. Le ${ }^{58} \mathrm{Ni}$, un des isotopes les plus réfringents, a un indice de 0.999998 pour une longueur d'onde de $1 \AA$.

Une des manifestations de la réfraction est le phénomène de réflexion totale lorsque les neutrons tombent sur une surface avec un angle d'incidence trop faible. Ils sont alors complètement réfléchis, sans perte d'intensité. Comme les indices sont très voisins de 1 , les angles critiques sont très petits.

On comprend bien ce phénomène de réflexion totale par de simples considérations énergétiques. Le neutron, qui interagit avec la matière par un potentiel $V(\overrightarrow{\mathbf{r}})$ verra en fait un potentiel moyen $U=\langle V(\vec{r})\rangle$. Son énergie cinétique totale est $\frac{\hbar^{2} \mathrm{k}^{2}}{2 \mathrm{~m}}$, mais la part de cette énergie qui lui permet de l'emporter sur $U$ et de pénétrer dans la matière est $\frac{\hbar^{2} \mathbf{k}_{\perp}^{2}}{2 \mathrm{~m}}$ où $\mathrm{k}_{\perp}$ est la composante perpendiculaire à la surface. Si cette énergie est inférieure à $U$, le faisceau de neutrons ne peut pas pénétrer la matière et subit la réflexion totale. Considérant que $\sin \theta=\frac{\mathrm{k}_{\perp}}{\mathrm{k}}$, l'angle critique est donné par

$$
\theta_{\mathrm{c}}=\frac{\lambda}{\mathrm{h}} \sqrt{2 \mathrm{mU}}
$$

Cet angle critique est donc proportionnel à la longueur d'onde. Si, pour $\lambda=1 \AA$, il ne vaut que 7 minutes d'angle dans le cas $d{ }^{58} \mathrm{Ni}$, il monte à plus de 2 degrés lorsque $\lambda$ dépasse $18 \AA$.

On utilise ces propriétés de réfraction et de réflexion totale pour mettre en oeuvre toute une optique instrumentale pour les faisceaux de neutrons avec guides, miroirs, multicouches et super miroirs. Bien entendu, c'est surtout avec des neutrons de grande longueur d'onde, pour des angles critiques les plus grands possibles, que l'optique neutronique est la plus performante.

\section{I-3 Les interactions neutron - matière}

II y a deux interactions différentes entre le neutron et les atomes qui forment la matière: l'interaction nucléaire et l'interaction magnétique.

\section{3. 1 L'interaction nucléaire (neutron - noyau)}

II existe des forces nucléaires entre le neutron et le noyau. Ces forces agissent sur des distances très courtes, de l'ordre de grandeur des dimensions du noyau, c'est à dire $10^{-4} \AA$.

\section{La longueur de Fermi}

Pour les neutrons utilisés en diffusion, dont la longueur d'onde est comprise entre une fraction d'angström et quelques angströms, on peut représenter le potentiel d'interaction par une fonction $\delta$

$$
\mathrm{V}_{\mathrm{N}}(\overrightarrow{\mathrm{r}})=\mathrm{a} \delta(\overrightarrow{\mathrm{r}})
$$




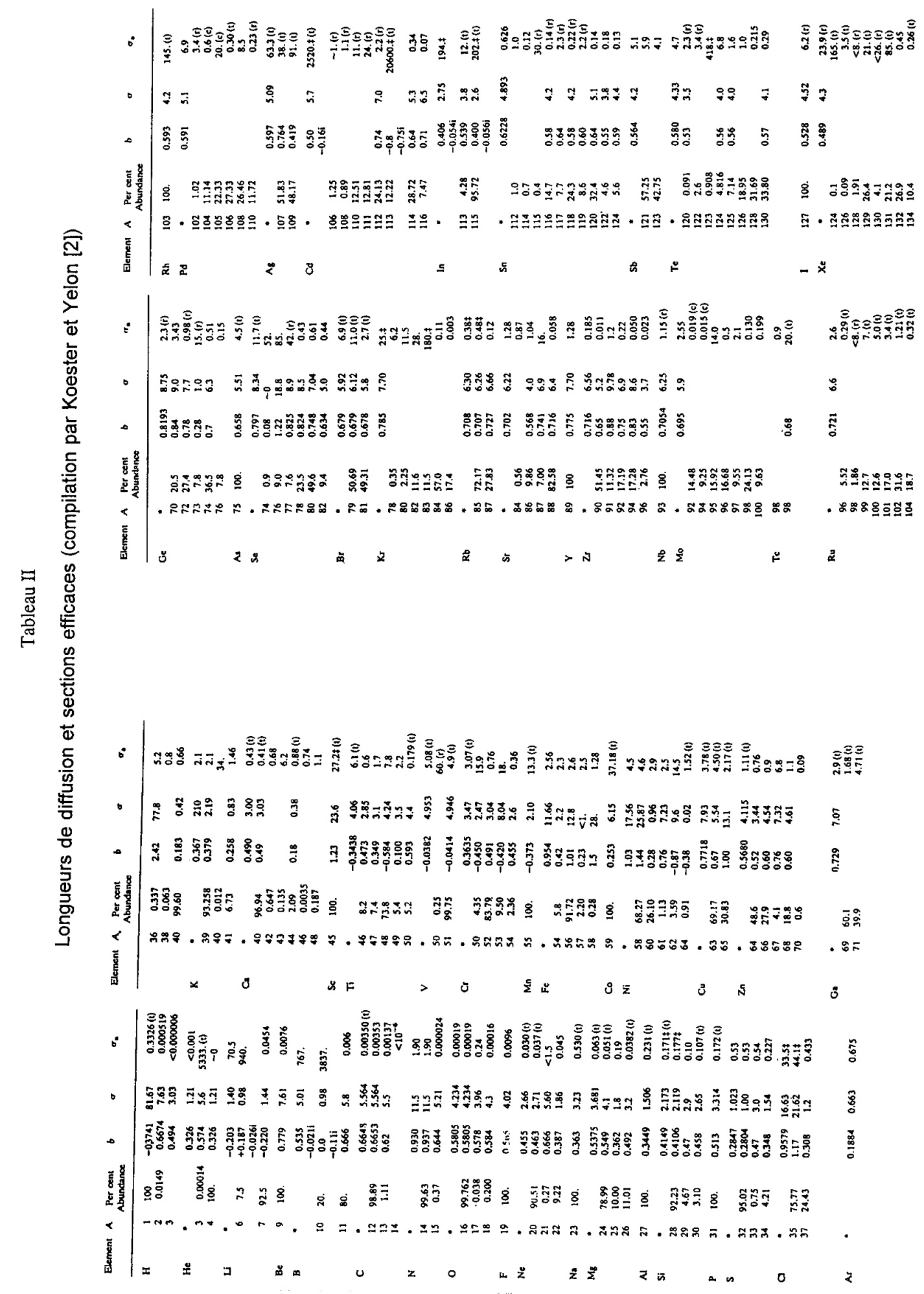




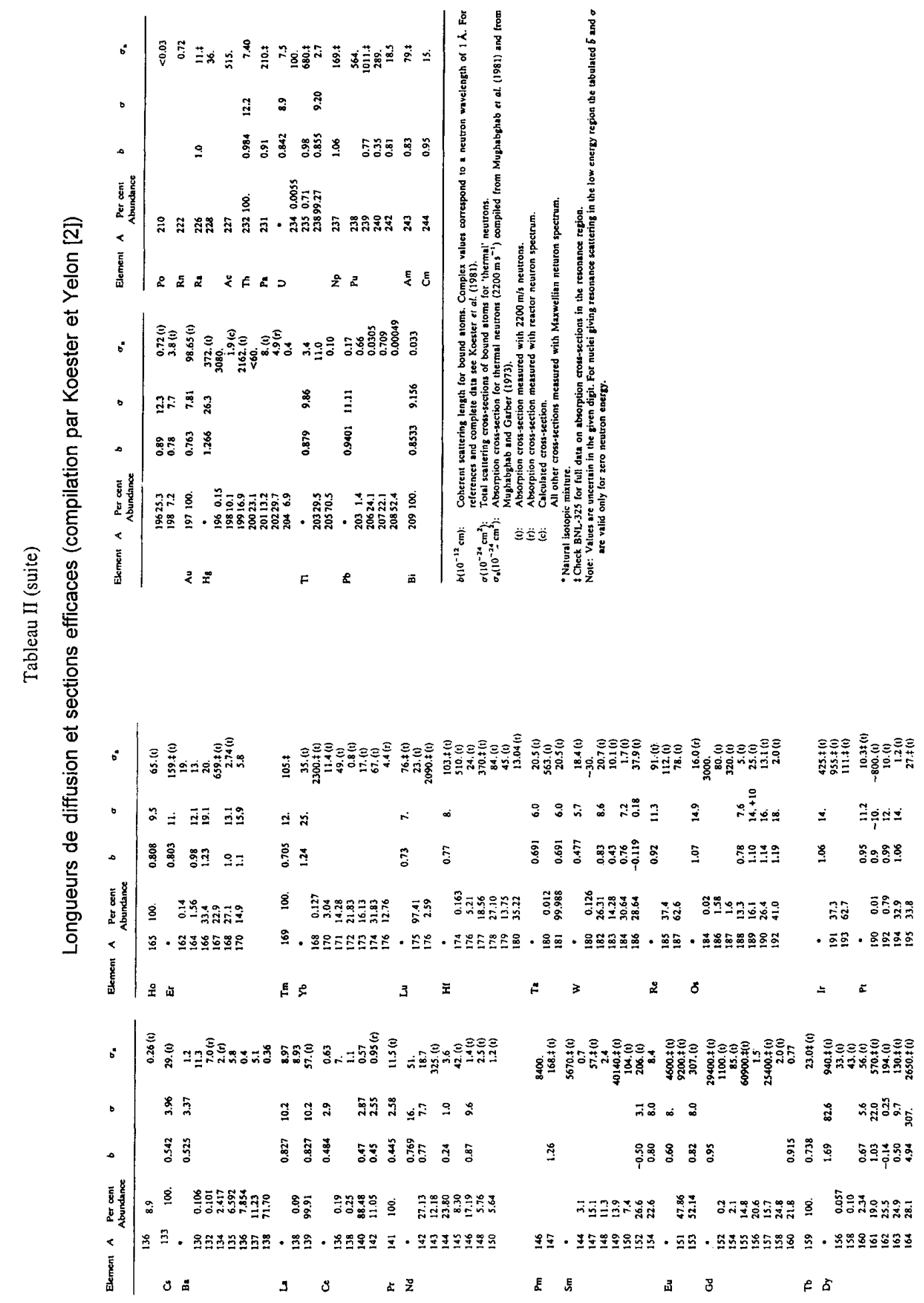


On a plutôt coutume de l'écrire sous la forme suivante, dite pseudo potentiel de Fermi:

$$
\mathrm{V}_{\mathrm{N}}(\overrightarrow{\mathrm{r}})=\frac{2 \pi \hbar^{2}}{\mathrm{~m}} \mathrm{~b} \delta(\overrightarrow{\mathrm{r}})
$$

Dans ces conditions, en revenant à la définition (14):

$$
\mathrm{f}\left(\overrightarrow{\mathrm{k}}, \overrightarrow{\mathrm{k}}^{\prime}\right)=\frac{\mathrm{m}}{2 \pi \hbar^{2}}\left\langle\overrightarrow{\mathrm{k}}^{\prime}\left|\mathrm{V}_{\mathrm{N}}\right| \overrightarrow{\mathrm{k}}\right\rangle=\mathrm{b}
$$

b est la longueur de diffusion ou longueur de Fermi, caractéristique de chaque noyau ${ }^{3}$. Pour un même élément, cette longueur est différente d'un isotope à l'autre.

La diffusion dépendant du spin

En réalité, la situation est plus compliquée que décrite ci-dessus car l'interaction neutron - noyau dépend du spin et cette dépendance intervient lorsque le noyau possède un spin nucléaire $I$.

Formellement, l'amplitude de diffusion nucléaire s'écrit

$$
f\left(\vec{k}, \vec{k}^{\prime}\right)=b+B \vec{\sigma} \cdot \vec{I}
$$

où l'opérateur de spin du neutron est $\frac{\hbar}{2} \vec{\sigma}$ et où l'opérateur vectoriel $\vec{\sigma}$ est représenté par les trois matrices de Pauli $\sigma_{\mathrm{x}}, \sigma_{\mathrm{y}}$ et $\sigma_{\mathrm{z}}$.

En fait, dès lors que le noyau possède un spin $(l \neq 0)$, il y a deux valeurs pour la longueur de diffusion. Selon que le spin total (noyau + neutron) prend la valeur $1+1 / 2$ ou $1-1 / 2$, la longueur de diffusion est $b_{+}$ou $b$.

Tableau III

Longueurs de diffusion dépendant du spin de quelques noyaux (en $\left.10^{-12} \mathrm{~cm}\right)$

\begin{tabular}{|llllll|}
\hline noyau & spin & $\overline{\mathrm{b}}$ & $\mathrm{b}_{+}$ & $\mathrm{b}$ & $\mathrm{b}_{+}-\mathrm{b}_{-}$ \\
\hline $1 \mathrm{H}$ & $1 / 2$ & -0.3741 & 1.085 & -4.750 & 5.835 \\
$2 \mathrm{H}$ & 1 & 0.6674 & 0.953 & 0.098 & 0.855 \\
$23 \mathrm{Na}$ & $3 / 2$ & 0.363 & 0.63 & -0.09 & 0.72 \\
$59 \mathrm{Co}$ & $7 / 2$ & 0.253 & -0.278 & 0.991 & -1.269 \\
\hline
\end{tabular}

\footnotetext{
${ }^{3}$ Cette longueur de diffusion considère que le noyau est fixe car fixement lié au système diffusant. S'il s'agit de noyaux complètement libres, cette longueur de diffusion est plus petite car le noyau, libre de bouger, recule pendant la collision. Le système se traite alors dans le système du centre de masse neutron-noyau et on arrive à une longueur de diffusion $b_{\text {free }}=b M /(m+M)$, où $m$ est la masse du neutron et $M$ la masse du noyau.
} 
Dans la pratique, même si les neutrons sont polarisés, les noyaux, sauf cas exceptionnel, ne le sont pas. Les neutrons sont diffusés au hasard, soit avec une longueur $b_{+}$soit avec une longueur $b$.. Pour calculer la moyenne, il faut prendre en compte le fait que la probabilité pour le spin du noyau composé de valoir $\mathrm{I}+\frac{1}{2}$ est $\frac{I+1}{2 I+1}$ tandis qu'elle est $\frac{I}{2 I+1}$ pour un spin $I-\frac{1}{2}$.

D'où

$$
\widetilde{b}=\frac{I+1}{2 I+1} b_{+}+\frac{I}{2 I+1} b_{-}
$$

Le tableau III donne la valeur des longueurs $b_{+}$ou $b$. et $\bar{b}$ de quelques isotopes. De tous les atomes, c'est pour le proton $\left({ }^{1} \mathrm{H}\right)$ que la différence entre $b_{+}$ou $b$. est la plus forte.

J'insiste sur le fait que, bien que l'interaction neutron - noyau dépende du spin, il s'agit bien d'interaction nucléaire et non pas d'une interaction magnétique avec le moment magnétique du noyau. Cette dernière existe, mais elle est négligeable: par rapport à l'interaction nucléaire ou à l'interaction magnétique avec les moments électroniques. Elle est plus faible de 3 ordres de grandeur.

\section{La moyenne sur les isotopes}

Chaque isotope ayant sa propre longueur de diffusion, et comme il n'y a pas de relation entre la nature de l'isotope et sa position, la longueur de diffusion moyenne d'un élément est donnée par une moyenne où interviennent les abondances isotopiques $c_{\mu}$ :

$$
\overline{\mathrm{b}}=\sum_{\text {isotopes }} \mathrm{c}_{\mu} \overline{\mathrm{b}}_{\mu}
$$

La tableau II, déjà cité pour les sections efficaces d'absorption, donne pour tous les éléments et pour un grand nombre d'isotopes les longueurs de diffusion $\bar{b}$ et $\bar{b}_{\mu}$.

\section{Longueurs de diffusion complexes et dépendant de la longueur d'onde}

Pour les noyaux qui ont un pic de résonance dans la gamme des longueurs d'onde utilisées (et qualifiés de cas pathologiques précédemment), il y a, au voisinage de la résonance, une contribution supplémentaire à la longueur de Fermi. Cette contribution est complexe: elle comprend une partie réelle $b^{\prime}$ et une partie imaginaire b". Toutes deux dépendent de la longueur d'onde et cette dépendance est donnée par les formules de Breit Wigner (Voir Marshall et Lovesey [3], Appendix A ou Mughabghab et al [4] qui donne tous les paramètres pour appliquer les formules de Breit Wigner).

\section{3. 2 L'interaction magnétique}

Associé à son spin, le neutron porte un moment magnétique $\mu_{\mathrm{n}}$ de $1.91 \mu_{\mathrm{BN}}$. Ce moment ressent tous les champs magnétiques de la matière et, en particulier, les champs émis par les moments magnétiques $\vec{M}$ des atomes dus aux couches électroniques incomplètes. Le couplage est alors le couplage dipôle-dipôle bien connu:

$$
V_{M}(\vec{r})=\frac{\vec{M} \cdot \vec{\mu}_{n}}{r^{3}}-\frac{3(\vec{M} \cdot \vec{r})\left(\vec{\mu}_{n} \cdot \vec{r}\right)}{r^{5}}
$$


ou encore

$$
\mathrm{V}_{\mathrm{M}}(\overrightarrow{\mathrm{r}})=-\vec{\mu}_{\mathrm{n}} \operatorname{rot}\left(\frac{\overrightarrow{\mathrm{M}} \wedge \overrightarrow{\mathrm{r}}}{\mathrm{r}^{3}}\right)
$$

Contrairement à l'interaction nucléaire, cette interaction est très anisotrope: elle dépend très fortement des orientations relatives de $\vec{\mu}_{n}, \vec{M}$ et $\vec{r}$.

En faisant usage des deux identités:

et

$$
\begin{aligned}
& \frac{\overrightarrow{\mathrm{r}}}{\mathrm{r}^{3}}=-\vec{\nabla}\left(\frac{1}{\mathrm{r}}\right) \\
& \frac{1}{\mathrm{r}}=\frac{1}{2 \pi^{2}} \int \frac{\mathrm{d} \overrightarrow{\mathrm{q}}}{\mathrm{q}^{2}} \mathrm{e}^{\mathrm{i} \overrightarrow{\mathrm{q}}}
\end{aligned}
$$

on arrive à l'amplitude de diffusion magnétique:

$$
\mathrm{f}\left(\overrightarrow{\mathrm{k}}, \overrightarrow{\mathrm{k}}^{\prime}\right)=\frac{\mathrm{m}}{2 \pi \hbar^{2}}\left\langle\overrightarrow{\mathrm{k}}^{\prime}\left|\mathrm{V}_{\mathrm{M}}\right| \overrightarrow{\mathrm{k}}\right\rangle=\mathrm{p} \vec{\sigma} \cdot \overrightarrow{\mathrm{M}}_{\perp} \mathrm{f}(\mathrm{Q})
$$

- où $p$ est un facteur qui vaut $0.269610^{-12} \mathrm{~cm} / \mu_{B}$ et représente l'amplitude de diffusion à $\mathrm{Q}=0$ pour un moment magnétique de $1 \mu_{\mathrm{B}}$.

- où $\vec{M}_{\perp}$ est la projection du moment magnétique de l'atome sur le plan perpendiculaire au vecteur de diffusion:

$$
\overrightarrow{\mathrm{M}}_{\perp}=\hat{\mathrm{Q}} \wedge(\overrightarrow{\mathrm{M}} \wedge \hat{\mathrm{Q}})=\overrightarrow{\mathrm{M}}-\hat{\mathrm{Q}}(\overrightarrow{\mathrm{M}} \cdot \hat{\mathrm{Q}})
$$

- avec $\hat{Q}$ vecteur unitaire selon le vecteur de diffusion $\left(\hat{Q}=\frac{\vec{Q}}{Q}\right)$

- et où le facteur de forme $f(Q)$ reflète l'extension spatiale du nuage électronique magnétique.

Figure 3: $\vec{M}_{\perp}$ projection de $\vec{M}$ sur le plan perpendiculaire au vecteur de diffusion:

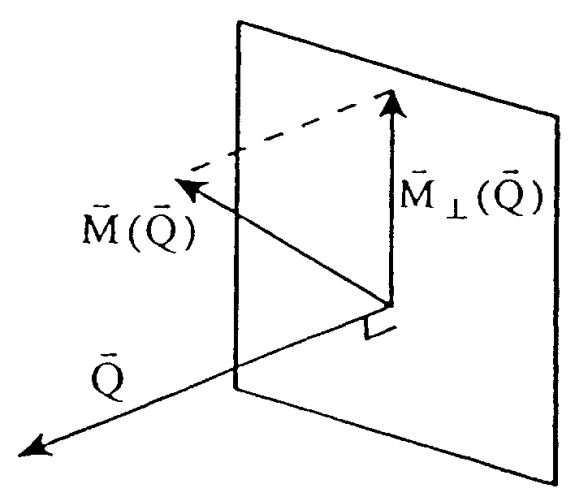




\section{I-4 Production de faisceaux de neutrons}

\section{4. 1 Production de neutrons}

Deux réactions sont utilisées en pratique pour produire des neutrons: la fission et la spallation.

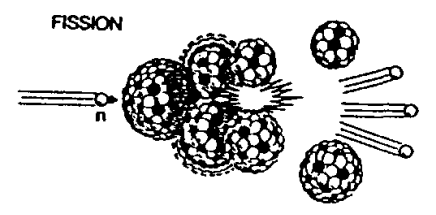

Figure 4: réactions de fission et de spallation

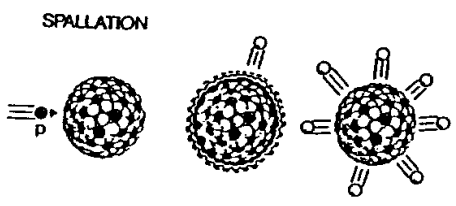

\section{Fission}

C'est la réaction qui se produit dans un réacteur (et dans une bombe atomique). Un neutron thermique frappe un noyau $235 \mathrm{U}$. Ce noyau devient très excité et se scinde en plusieurs fragments avec émission d'un nombre variable de neutrons: entre 2 et 5 , en moyenne 2,5. L'énergie moyenne de ces neutrons est $2 \mathrm{MeV}$.

\section{Spallation}

Un proton de haute énergie, issu d'un accélérateur, frappe une cible composée d'atomes lourds (U, W, Hg..). Ces noyaux lourds éjectent alors un certain nombre de particules dont une vingtaine de neutrons ayant une énergie de quelques $\mathrm{MeV}$.

Les jets de protons sur la cible peuvent être pulsés (ISIS) ou continus (SINQ).

\section{4. 2. Modération des neutrons}

Les expériences de diffusion neutronique se font avec des neutrons dont l'énergie va d'une fraction de meV à quelques meV. Avec, au départ des neutrons de quelques $\mathrm{MeV}$, il y a 9 ordres de grandeur à perdre pour pouvoir les utiliser. Cette perte d'énergie s'effectue par collisions avec les noyaux d'un modérateur. Plus ces noyaux sont légers, plus le choc est inélastique et plus efficace est le processus de perte d'énergie. Les éléments les plus utilisés sont l'hydrogène (l'eau), le deutérium (l'eau lourde), le béryllium et le graphite. Après quelques dizaines de collisions, les neutrons sont en équilibre thermique avec le milieu. II faut pour cela quelques centimètres d'eau ou quelques décimètres d'eau lourde.

Des neutrons en équilibre thermique avec un modérateur à très basse température comme un dewar plein d'hydrogène ou de deutérium liquide (source froide) sont des neutrons de basse énergie (inférieure à $10 \mathrm{meV}$ ) ou neutrons froids. Des neutrons en équilibre thermique avec de l'eau à température ambiante sont dits neutrons thermiques (énergie typique $25 \mathrm{meV}$ ). Des neutrons en équilibre thermique avec un bloc de graphite chauffé à $2000^{\circ} \mathrm{C}$ (source chaude) sont des neutrons chauds 
(énergie supérieure à $100 \mathrm{meV}$ ). Des spectres caractéristiques des différentes sources sont représentés sur la figure 5 .

Figure 5: Spectres caractéristiques des différentes sources

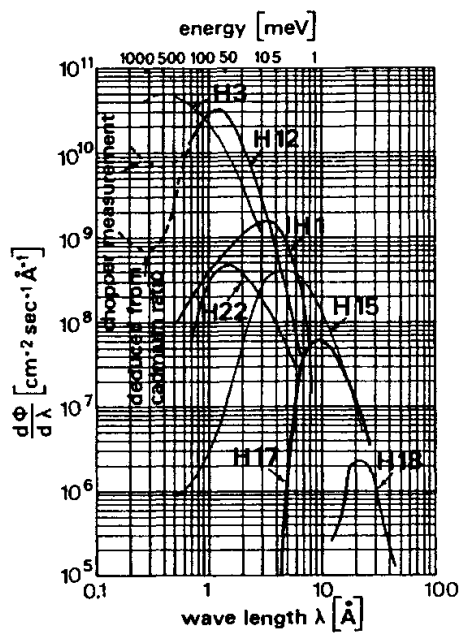

\section{4. 3. Extraction des faisceaux}

On obtient un faisceau de neutrons en ouvrant un canal dans la protection et dans le modérateur, trou par lequel les neutrons bien situés et bien dirigés peuvent s'échapper (voir figure 6). Ces neutrons ont donc la direction imposée par le canal. Leurs vitesses (ou leurs longueurs d'onde) sont réparties selon le spectre maxwellien correspondant à la température du modérateur.

Figure 6: Disposition des sources et des canaux à I'ILL

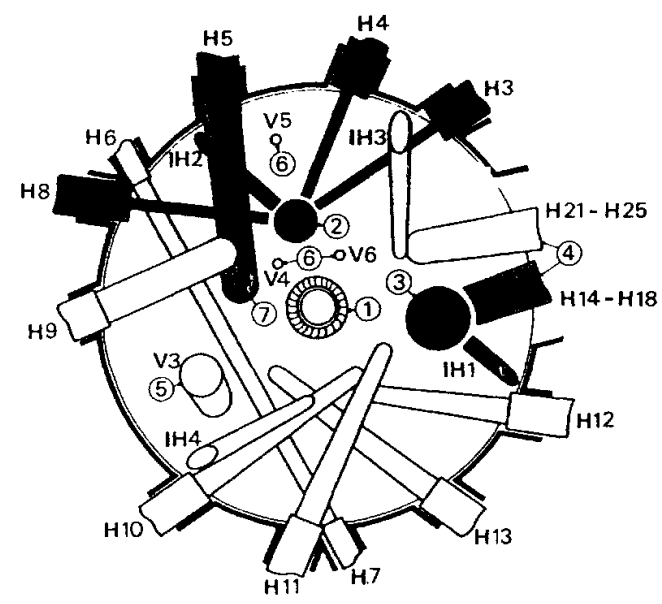




\section{I-5 Détection des neutrons}

Le neutron n'ayant pas de charge, il faut pour le détecter lui faire subir une réaction nucléaire et détecter soit les particules chargées soit les photons émis par cette réaction.

\section{5. 1. Compteurs}

\section{Compteurs proportionnels}

Ils sont constitués d'une chambre à gaz contenant le gaz absorbant. II s'agit soit de $\mathrm{BF}_{3}$ enrichi en ${ }^{10} \mathrm{~B}$ :

soit de ${ }^{3} \mathrm{He}$ :

$$
\mathrm{n}+{ }^{10 \mathrm{~B}} \rightarrow{ }^{7} \mathrm{Li}+{ }^{4} \mathrm{He}+\gamma
$$

$$
\mathrm{n}+{ }^{3} \mathrm{He} \rightarrow \mathrm{p}+{ }^{3} \mathrm{H}
$$

Les particules chargées émises par la réaction nucléaire ionisent le gaz. Les électrons ainsi émis sont accélérés par les électrodes (voltage de 2 ou $3 \mathrm{kV}$ ) et ionisent encore plus d'atomes de gaz. II en résulte une décharge sur le fil d'anode. les photons $\gamma$ créent des impulsions beaucoup plus faibles et faciles à éliminer.

\section{Scintillateurs}

Les neutrons sont absorbés dans une couche de verre ou de plastique chargée en $6 \mathrm{Li}$ et $\mathrm{ZnS}$.

$$
\mathrm{n}+{ }^{6} \mathrm{Li} \rightarrow{ }^{3} \mathrm{H}+{ }^{4} \mathrm{He}
$$

Les particules chargées créent des excitations électroniques qui se désactivent en donnant des photons. Ces photons sont transportés par un guide de lumière vers un photomultiplicateur.

Les scintillateurs ont une zone d'absorption des neutrons moins épaisse que les chambres à gaz. Ils sont de ce fait plus précis pour les expériences de temps de vol.

\section{Chambres à fission}

Une feuille d'uranium solide est fixée dans une chambre à gaz. Les particules émises dans la réaction de fission sont comptées dans la chambre à gaz.

\section{5. 2. Multidétecteurs}

Plutôt que de déplacer un compteur unique dans toutes les directions de l'espace

(a)

resistive wire gas detector
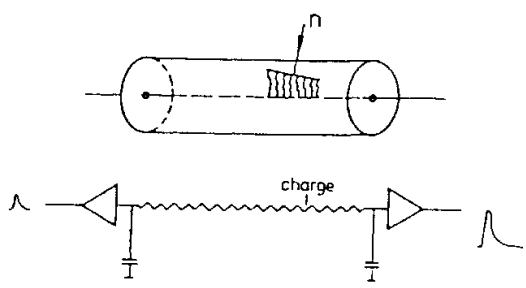

(b)

banana: a multiwire gas detector

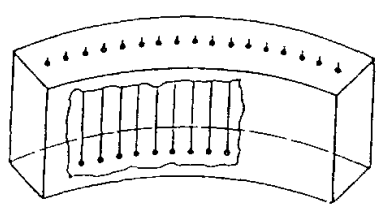

Figure 7: PSD à une dimension: (a) à fil résistif, (b) banane multifils 
pour compter les neutrons diffusés, il est beaucoup plus rentable de tapisser l'espace de surfaces de détection sensibles.

La première possibilité a été d'utiliser un certain nombre de détecteurs individuels. Puis sont apparus les détecteurs sensibles à la position (Position Sensitive Detector ou PSD) à une ou deux dimensions.

A une dimension on peut utiliser un fil résistif qui permet de localiser la position où le neutron a été absorbé (voir figure $7 a$ ) ou bien un grand nombre de fils parallèles comme sur la banane de la figure $7 \mathrm{~b}$. A deux dimensions le détecteur implique 2 systèmes de fils, perpendiculaires, qui permettent une localisation en $x$ et en y (voir figure 8 ).

Figure 8: PSD à deux dimensions

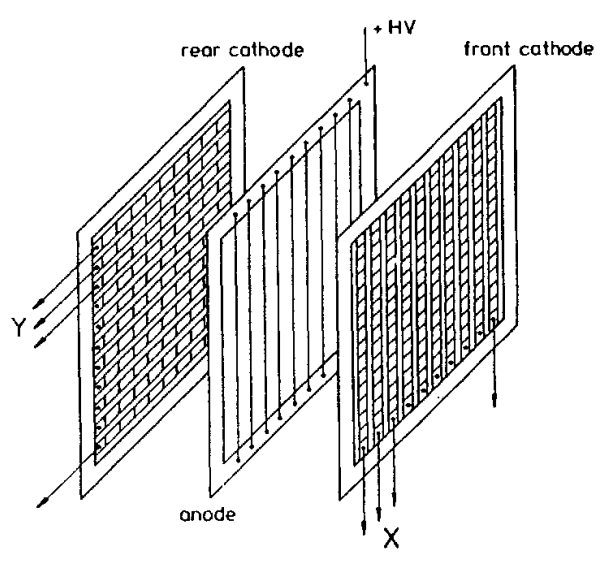

L'usage des multidétecteurs s'est généralisé au cours des deux dernières décennies car c'est un moyen d'augmenter l'efficacité d'un instrument de façon beaucoup plus économique que d'augmenter le flux de la source de neutrons.

\section{La diffusion de neutrons: un corréloscope dans l'espace $(\overrightarrow{\mathrm{Q}}, \omega)$}

On va voir dans ce chapitre ce que mesure la diffusion de neutrons et comment les mesures sont faites.

\section{II-1 La mesure des neutrons diffusés: une mesure dans l'espace $(\overrightarrow{\mathbf{Q}}, \omega)$, transformé de Fourier de l'espace $(\overrightarrow{\mathbf{r}}, \mathbf{t})$}

Dans ce chapitre nous allons esquisser, sans entrer dans les détails, les étapes qui mènent à l'expression de la section efficace de diffusion. Nous suivrons plus particulièrement la diffusion par les noyaux et donnerons par analogie les résultats pour la diffusion par les moments magnétiques.

\section{1. 1. Le phénomène de diffusion}

Au cours de la diffusion, le neutron passe d'un état défini par le vecteur d'ondes $\vec{k}$ à un état défini par le vecteur d'onde $\overrightarrow{\mathrm{k}}^{\prime}$. Pendant le même temps, le système diffusant passe d'un état $\lambda$ à un état $\lambda^{\prime}$. 


\section{Règle d'or de Fermi}

La section efficace différentielle de diffusion est donnée par la règle d'or de Fermi qui donne la probabilité de transition d'un état caractérisé par les deux variables $\mid \vec{k}, \lambda>$ vers un état caractérisé par $\mid \vec{k}^{\prime}, \lambda^{\prime}>4$.

$$
\left(\frac{\mathrm{d} \sigma}{\mathrm{d} \Omega}\right)_{\substack{\mathrm{k} \rightarrow \overrightarrow{\mathrm{k}}^{\prime} \\ \lambda \rightarrow \lambda^{\prime}}}=\frac{\mathrm{k}^{\prime}}{\mathrm{k}}\left(\frac{\mathrm{m}}{2 \pi \hbar^{2}}\right)^{2}\left|\left\langle\overrightarrow{\mathrm{k}}^{\prime}, \lambda^{\prime}|\mathrm{v}| \overrightarrow{\mathrm{k}}, \lambda\right\rangle\right|^{2}
$$

où $V$ est le potentiel d'interaction entre le neutron et le système.

\section{Conservation de l'énergie}

En considérant que l'énergie de l'ensemble (neutron + système) ne doit pas changer pendant le processus de diffusion,

$$
\mathrm{E}+\mathrm{E}_{\lambda}=\mathrm{E}^{\prime}+\mathrm{E}_{\lambda^{\prime}}
$$

on peut alors exprimer la section efficace différentielle partielle:

$$
\left(\frac{\mathrm{d}^{2} \sigma}{\mathrm{d} \Omega \mathrm{dE} \mathrm{E}^{\prime}}\right)_{\substack{\overrightarrow{\mathrm{k}} \rightarrow \vec{k}^{\prime} \\ \lambda \rightarrow \lambda^{\prime}}}=\frac{\mathrm{k}^{\prime}}{\mathrm{k}}\left(\frac{\mathrm{m}}{2 \pi \hbar^{2}}\right)^{2}\left|\left\langle\overrightarrow{\mathrm{k}}^{\prime}, \lambda^{\prime}|\mathrm{V}| \overrightarrow{\mathrm{k}}, \lambda\right\rangle\right|^{2} \delta\left(\mathrm{E}^{\prime}+\mathrm{E}_{\lambda^{\prime}}-\mathrm{E}-\mathrm{E}_{\lambda}\right)
$$

\section{Transformée de Fourier de la fonction potentiel}

Le potentiel d'interaction entre le neutron et le système diffuseur est la somme des potentiels d'interaction entre le neutron et les différents centres atomiques $\mathbf{j}$ :

$$
V(\overrightarrow{\mathbf{r}})=\sum_{j} V_{j}\left(\vec{r}_{j}\right)=\sum_{j} V_{j}\left(\vec{r}-\vec{R}_{j}\right)
$$

Où $\overrightarrow{\mathrm{r}}$ est la coordonnée absolue du neutron, $\overrightarrow{\mathrm{R}}_{j}$ celle de l'atome $\mathrm{j}$ et $\vec{r}_{\mathrm{j}}=\overrightarrow{\mathrm{r}}-\overrightarrow{\mathrm{R}}_{\mathrm{j}}$ la coordonnée du neutron par rapport à l'atome $\mathrm{j}$. L'intégration sur $\overrightarrow{\mathrm{r}}$ donne :

$$
\left\langle\vec{k}^{\prime}, \lambda^{\prime}|v| \vec{k}, \lambda\right\rangle=\sum_{j} V_{j}(\vec{Q})\left\langle\lambda^{\prime}\left|e^{i \vec{Q} \cdot \vec{k}_{j}}\right| \lambda\right\rangle
$$

avec $\vec{Q}=\vec{k}-\vec{k}^{\prime}$, vecteur de diffusion, $V_{j}(\vec{Q})$ étant la transformée de Fourier du potentiel $V_{j}\left(\vec{r}_{\mathrm{j}}\right)$

$$
V_{j}(\vec{Q})=\int e^{i \vec{Q}^{-} \vec{r}_{j}} V_{j}\left(\vec{r}_{j}\right) d \vec{r}_{j}
$$

\footnotetext{
${ }^{4}$ En fait, le neutron est aussi caractérisé par sa variable de spin qui, au cours de la diffusion, passe de la valeur $\sigma$ à la valeur $\sigma^{\prime}$. En conséquence, la formule (34) ci-dessus doit être remplacée par la formule suivante :$$
\left(\frac{\mathrm{d} \sigma}{\mathrm{d} \Omega}\right)_{\substack{\overrightarrow{\mathrm{k}} \rightarrow \overrightarrow{\mathrm{k}}^{\prime} \\ \sigma \rightarrow \lambda^{\prime} \\ \sigma \rightarrow \sigma^{\prime}}}=\frac{\mathrm{k}}{\mathrm{k}^{\prime}}\left(\frac{\mathrm{m}}{2 \pi \hbar^{2}}\right)^{2}\left|\left\langle\overrightarrow{\mathrm{k}}^{\prime}, \lambda^{\prime}, \sigma^{\prime}|\mathrm{V}| \overrightarrow{\mathrm{k}}, \lambda, \sigma\right)\right|^{2} .
$$

Dans le cadre de cette introduction, par soucis de simplification, nous ne traiterons pas la variable de spin. Les résultats donnés dans ce chapitre ne seront exacts que pour la diffusion par des neutrons non polarisés.
} 


\section{1. 2. Diffusion par les noyaux}

Comme potentiel $\mathrm{V}_{\mathrm{j}}\left(\overrightarrow{\mathrm{r}}_{\mathrm{j}}\right)$, nous prenons le pseudo-potentiel de Fermi:

$$
\mathrm{V}_{\mathrm{j}}\left(\overrightarrow{\mathrm{r}}_{\mathrm{j}}\right)=\frac{2 \pi \hbar^{2}}{\mathrm{~m}} \mathrm{~b}_{\mathrm{j}} \delta\left(\overrightarrow{\mathrm{r}}_{\mathrm{j}}\right)
$$

ce qui nous donne comme transformée de Fourier du potentiel :

$$
\mathrm{V}_{\mathrm{j}}(\overrightarrow{\mathrm{Q}})=\frac{2 \pi \hbar^{2}}{\mathrm{~m}} b_{\mathrm{j}}
$$

et pour la section efficace différentielle partielle:

$$
\left(\frac{\mathrm{d}^{2} \sigma}{\mathrm{d} \Omega \mathrm{dE}^{\prime}}\right)_{\substack{\hat{k} \rightarrow \overrightarrow{\mathrm{k}}^{\prime} \\ \lambda \rightarrow \lambda^{\prime}}}=\frac{\mathrm{k}^{\prime}}{\mathrm{k}}\left|\left\langle\lambda^{\prime}\left|\sum_{\mathrm{j}} \mathrm{b}_{\mathrm{j}} \mathrm{e}^{\mathrm{i} \cdot \overrightarrow{\mathrm{Q}} \cdot \overline{\mathrm{k}}_{j}}\right| \lambda\right\rangle\right|^{2} \delta\left(\mathrm{E}^{\prime}+\mathrm{E}_{\lambda^{\prime}}-\mathrm{E}-\mathrm{E}_{\lambda}\right)
$$

Représentation intégrale de la fonction de Dirac pour l'énergie

On utilise la forme intégrale de la fonction de Dirac $\delta$ pour l'énergie :

$$
\delta(\gamma)=\frac{1}{2 \pi} \int_{-\infty}^{+\infty} \mathrm{e}^{\mathrm{i} \gamma} \mathrm{dt}
$$

Avec $E-E^{\prime}=\hbar \omega$, l'énergie cédée au système par le neutron :

$$
\delta\left(\mathrm{E}^{\prime}+\mathrm{E}_{\lambda^{\prime}}-\mathrm{E}-\mathrm{E}_{\lambda}\right)=\delta\left(\mathrm{E}_{\lambda^{\prime}}-\mathrm{E}_{\lambda}-\hbar \omega\right)=\frac{1}{2 \pi \hbar} \int_{-\infty}^{+\infty} \mathrm{e}^{\mathrm{i}\left(\mathrm{E}_{\lambda^{\prime}} \cdot \mathrm{E}_{\lambda}\right) \frac{1}{h}} \mathrm{e}^{-\mathrm{i} \omega t} \mathrm{dt}
$$

Les états $\underline{|\lambda\rangle}$ et $\left|\lambda^{\prime}\right\rangle$ ont comme valeurs propres $\underline{E_{\lambda}}$ et $\underline{E_{\lambda^{\prime}}}$

II en résulte les relations suivantes :

$$
\begin{array}{ll}
H|\lambda\rangle=E_{\lambda}|\lambda\rangle & H\left|\lambda^{\prime}\right\rangle=E_{\lambda^{\prime}}\left|\lambda^{\prime}\right\rangle \\
e^{-i H \frac{t}{h}}|\lambda\rangle=e^{-i E_{\lambda} \frac{t}{h}}|\lambda\rangle & e^{-i H \frac{t}{h}}\left|\lambda^{\prime}\right\rangle=e^{-i E_{2} \cdot \frac{t}{h}}\left|\lambda^{\prime}\right\rangle
\end{array}
$$

et pour la section efficace :

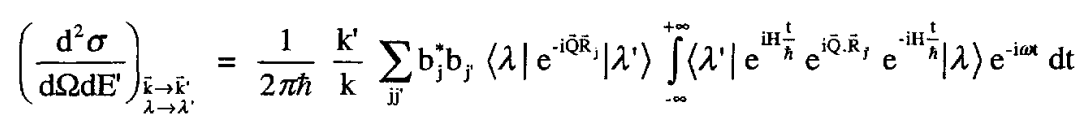

Somme sur $\underline{\left|\lambda^{\prime}\right\rangle}$ et moyenne sur $\underline{|\lambda\rangle}$

Dans une expérience de diffusion neutronique, on ne se limite pas à la section efficace liée à la transition d'un système qui passe de l'état $\mid \lambda>$ à l'état $|\lambda|>$.

-Pour un état initial $\mid \lambda>$, on fait la somme des sections efficaces correspondant à tous les états finaux $\left|\lambda^{\prime}\right\rangle$ du système. 
-D'autre part on fait une somme sur tous les états initiaux $\mid \lambda>$ possibles, en tenant compte de la probabilité $\mathrm{p}_{\lambda}$ pour le système de se trouver dans cet état initial, compte tenu de sa température.

On applique la relation de fermeture :

$$
\sum_{\lambda^{\prime}}\left\langle\lambda|\mathrm{A}| \lambda^{\prime}\right\rangle\left\langle\lambda^{\prime}|\mathrm{B}| \lambda\right\rangle=\langle\lambda|\mathrm{AB}| \lambda\rangle
$$

et on utilise l'opérateur de Heisenberg dépendant du temps :

$$
\vec{R}_{j^{\prime}}(t)=e^{i H \frac{t}{\hbar}} \vec{R}_{j^{\prime}} e^{-i H \frac{t}{\hbar}}
$$

avec

$$
\overrightarrow{\mathrm{R}}_{\mathrm{j}}(0)=\overrightarrow{\mathrm{R}}_{\mathrm{j}}
$$

et on arrive à l'expression de la section efficace différentielle pour la diffusion nucléaire :

$$
\left(\frac{d^{2} \sigma}{d \Omega d E^{\prime}}\right)_{\vec{k} \rightarrow \vec{k}^{\prime}}^{N}=\frac{1}{2 \pi \hbar} \frac{k^{\prime}}{k} \sum_{j j^{\prime}}^{+\infty} \int_{-\infty}^{+\infty}\left\langle b_{j}^{*} b_{j^{\prime}} e^{-i \vec{Q} \bar{R}_{j}(0)} e^{i \bar{Q} \vec{R}_{f}(t)}\right\rangle e^{-i \omega x} d t
$$

où la valeur entre crochets $\langle A\rangle$ représente la moyenne thermique de l'opérateur $A$ à la température $\mathrm{T}$, moyenne donnée par la formule:

$$
\langle\mathrm{A}\rangle=\sum_{\lambda^{\prime}} \mathrm{p}_{\lambda^{\prime}}\left\langle\lambda^{\prime}|\mathrm{A}| \lambda^{\prime}\right\rangle
$$

Cette expression (49) de la section efficace différentielle partielle est une fonction du vecteur de diffusion $\overrightarrow{\mathrm{Q}}$ et du transfert d'énergie $\hbar \omega$

Si on définit un opérateur "densité" nucléaire" $\hat{\rho}(\overrightarrow{\mathrm{r}}, \mathrm{t})$ comme une densité de noyaux pondérée par les longueurs de Fermi de chaque noyau :

$$
\hat{\rho}(\overrightarrow{\mathrm{r}}, \mathrm{t})=\sum_{\mathrm{j}} \mathrm{b}_{\mathrm{j}} \delta\left(\overrightarrow{\mathrm{r}}-\overrightarrow{\mathrm{R}}_{\mathrm{j}}(\mathrm{t})\right)
$$

la section efficace différentielle partielle est la double transformée de Fourier de la fonction de corrélation de cette densité nucléaire avec elle-même.

$$
\left(\frac{\mathrm{d}^{2} \sigma}{\mathrm{d} \Omega \mathrm{dE} E^{\prime}}\right)^{\mathrm{N}}=\mathrm{DTF} \mathrm{P}[\hat{\rho}(\overrightarrow{\mathrm{r}}, \mathrm{t}) \mid \hat{\rho}(\overrightarrow{0}, 0)]
$$

Cette notion d'autocorrélation est expliquée en termes de probabilités dans le paragraphe suivant.

\section{1. 3. Un corréloscope dans l'espace $(\overrightarrow{\mathbf{r}}, \mathbf{t})$}

Pour comprendre ce que représente la fonction de corrélation, nous allons donner l'exemple développé par Scherm et Fak [5] sur le "corréloscope à mouches". Des mouches volent dans une pièce qui représente notre espace direct ou espace $(\vec{r}, t)$. Nous voulons les étudier à l'aide d'un corréloscope constitué de 2 viseurs $A$ et $B$ reliés par une règle graduée et par un chronomètre (figure 9). 
Figure 9: un corréloscope à mouches, d'après [5]
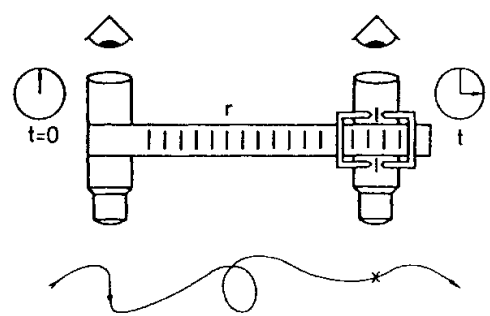

On commence par chercher une mouche dans le viseur A. Quand on en voit une, on met la règle à zéro en $\mathrm{A}$ et on déclanche le chronomètre. On se met alors à chercher une mouche dans le viseur B. Quand on en voit une, la même ou une autre, on note sa coordonnée d'espace $\overrightarrow{\mathrm{r}}$ et sa coordonnée de temps t. On recommence l'opération un très grand nombre de fois (d'abord le viseur A, puis le viseur B). On a alors mesuré $\mathrm{P}[\rho(\overrightarrow{\mathrm{r}}, \mathrm{t}) \mid \rho(\overrightarrow{0}, 0)]$, la probabilité conditionnelle d'avoir une mouche en $(\overrightarrow{\mathrm{r}}, \mathrm{t})$ si on en a vu une en $(\overrightarrow{0}, 0)$.

C'est la fonction de l'espace réel que l'on cherche à déterminer par la diffusion de neutrons: la probabilité conditionnelle d'avoir une particule en $(\vec{r}, t)$ s'il en existe une en $(\overrightarrow{0}, 0)$.

En fait les neutrons ne mesurent pas directement cette fonction de corrélation, mais une fonction de $(\overrightarrow{\mathrm{Q}}, \omega)$ donnée par la formule (49), qui est sa double transformée de Fourier par rapport à l'espace et au temps.

\section{1. 4. Section efficace pour la diffusion magnétique}

Pour la diffusion magnétique, la formule (49) s'applique, avec $b_{j}$ remplacé par $p \vec{\sigma} \cdot \vec{M}_{\perp j} f_{j}(Q)$

où $\mathrm{p}=0.269610^{-12} \mathrm{~cm} / \mu_{\mathrm{B}}$ est une constante

$\overrightarrow{\mathrm{M}}_{\perp \mathrm{j}}$ est la projection du moment magnétique $\overrightarrow{\mathrm{M}}_{\mathrm{j}}$ sur le plan perpendiculaire à $\vec{Q}$ $\mathrm{f}_{\mathrm{j}}(\mathrm{Q})$ est le facteur de forme du nuage magnétique de l'atome $\mathrm{j}$

$\vec{\sigma}$ est l'opérateur vectoriel "matrices de Pauli" du neutron $\left(\vec{s}=\frac{\hbar}{2} \vec{\sigma}\right)$

Ce qui donne, pour des neutrons non polarisés:

$$
\left(\frac{d^{2} \sigma}{d \Omega d E^{\prime}}\right)_{\vec{k} \rightarrow \bar{k}^{\prime}}^{M}=\frac{p^{2}}{2 \pi \hbar} \frac{k^{\prime}}{k} \sum_{j j^{\prime}} \int_{-\infty}^{+\infty}\left\langle\vec{M}_{l j}^{*}(0) \cdot \vec{M}_{\perp j^{\prime}}(t) f_{j} f_{j^{\prime}} e^{-i \vec{Q} \cdot \overrightarrow{\mathbf{R}}_{j}(0)} e^{i \vec{Q} \cdot \overrightarrow{\mathbf{R}}_{f}(t)}\right\rangle \mathrm{e}^{-i \omega t} d t
$$

Il s'agit là encore d'une fonction dans l'espace $(\vec{Q}, \omega)$ qui est la double transformée de Fourier par rapport à l'espace et au temps de la fonction de corrélation magnétique avec elle même:

$$
\left(\frac{d^{2} \sigma}{d \Omega d E^{\prime}}\right)^{M}=\operatorname{DTFP} P\left[\vec{m}_{\perp}(\vec{r}, t) \mid \vec{m}_{\perp}(\overrightarrow{0}, 0)\right]
$$

où $\overrightarrow{\mathrm{m}}(\overrightarrow{\mathrm{r}}, \mathrm{t})$ représente la densité d'aimantation, fonction de l'espace et du temps. 


\section{1. 5. Section efficace totale}

Avec des neutrons non polarisés, il n'y a pas d'interférence entre les amplitudes nucléaires et les amplitudes magnétiques. La section efficace totale est la somme de la section efficace nucléaire et de la section efficace magnétique :

$$
\left(\frac{d^{2} \sigma}{d \Omega d E^{\prime}}\right)^{\text {Tot }}=\left(\frac{d^{2} \sigma}{d \Omega d E^{\prime}}\right)^{N}+\left(\frac{d^{2} \sigma}{d \Omega d E^{\prime}}\right)^{M}
$$

\section{II-2 Sections efficaces cohérentes et incohérentes}

C'est là une particularité très générale et très importante de la diffusion des neutrons qui est due au fait que les différents atomes d'un même élément diffusent les neutrons avec des longueurs de diffusions qui ne sont pas les mêmes d'un atome à l'autre. Ce phénomène existe pour quelques cas de figure dans la diffusion des rayons $\mathrm{X}$, mais n'a pas le caractère systématique que nous rencontrons ici.

En diffusion nucléaire, la différence de longueurs de diffusion provient d'une part de la présence d'isotopes qui ont chacun leur propre longueur de Fermi, et d'autre part, dans le cas d'isotopes ayant un spin nucléaire, de l'existence de deux possibilités: $b_{+}$ ou b. comme longueur de diffusion.

Pour simplifier l'exposé, on va supposer dans ce qui suit que le système est monoatomique et que sa longueur de Fermi est réelle. Comme tous les atomes sont chimiquement équivalents, il n'y a pas de relation entre la valeur de $b_{j}$ et sa position $\vec{R}_{\mathbf{j}}$ :

$$
\left\langle b_{j} b_{j}, e^{-i \vec{Q} \cdot \hat{R}_{j}(0)} e^{\mathrm{i} \vec{Q} \cdot \vec{R}_{j}(t)}\right\rangle=\left\langle b_{j} b_{j j}\right\rangle\left\langle e^{-i \bar{Q} \cdot \vec{R}_{j}(0)} e^{i \bar{Q} \cdot \vec{R}_{j}(t)}\right\rangle
$$

La formule (49) s'écrit alors:

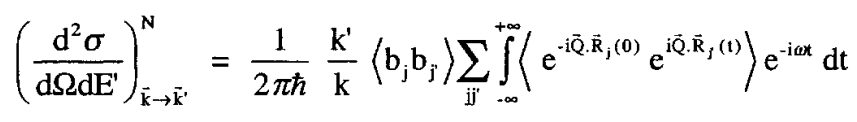

Intéressons-nous à $\left\langle b_{j} b_{j^{\prime}}\right\rangle$

Pour chaque atome, écrivons que:

$$
b_{j}=\bar{b}+\Delta b_{j}
$$

où $\Delta b_{j}$ représente l'écart à la moyenne, ce qui implique que sa propre moyenne est nulle : $\left\langle\Delta \mathrm{b}_{\mathrm{j}}\right\rangle=0$

On va devoir évaluer la moyenne des produits $b_{j} b_{j^{\prime}}$ pris sur tous les couples jj'

$$
\begin{aligned}
\left\langle b_{j} b_{j^{\prime}}\right\rangle & =\left\langle\left(\bar{b}+\Delta b_{j}\right)\left(\bar{b}+\Delta b_{j^{\prime}}\right)\right\rangle \\
& =\left\langle(\bar{b})^{2}+\bar{b} \Delta b_{j}+\bar{b} \Delta b_{j^{\prime}}+\left(\Delta b_{j}\right)\left(\Delta b_{j^{\prime}}\right)\right\rangle
\end{aligned}
$$

Les moyennes du deuxième et du troisième terme sont nulles compte tenu de (58). 
Quant à la moyenne sur $\left(\Delta \mathrm{b}_{\mathrm{j}}\right)\left(\Delta \mathrm{b}_{\mathrm{j}^{\prime}}\right)$, il faut distinguer:

$$
\begin{array}{rlrl}
\left\langle\left(\Delta b_{j}\right)\left(\Delta b_{j^{\prime}}\right)\right\rangle & =0 & \text { si j } \neq j \\
& =\overline{(\Delta b)^{2}} \quad \text { si } j^{\prime}=j
\end{array}
$$

et la section efficace différentielle partielle se sépare en deux parties :

$$
\begin{aligned}
& \left(\frac{d^{2} \sigma}{d \Omega d E^{\prime}}\right)_{\vec{k} \rightarrow \vec{k}^{\prime}}^{N}=\frac{1}{2 \pi \hbar} \frac{k^{\prime}}{k}(\bar{b})^{2} \sum_{i i^{\prime}}^{+\infty} \int_{-\infty}^{+\infty}\left\langle e^{-i \vec{Q} \cdot \vec{R}_{j}(0)} e^{i \vec{Q} \cdot \vec{R}_{j}(t)}\right\rangle e^{-i \omega t} d t
\end{aligned}
$$

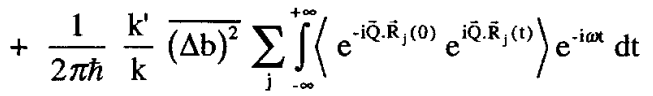

- une partie "cohérente" qui représente l'interaction entre paires d'atomes $\left(\sum_{\mathrm{j} j^{\prime}}\right)$, construite avec la moyenne du carré des longueurs de Fermi $(\overline{\mathrm{b}})^{2}$

- une partie "incohérente" qui représente l'interaction de chaque atome avec lui-même $\left(\sum_{\mathrm{j}}\right)$, construite avec la moyenne du carré de l'écart à la moyenne $\overline{(\Delta \mathrm{b})^{2}}$

Autre expression pour la moyenne du carré de l'écart $\overline{(\Delta b)^{2}}$

$$
\begin{gathered}
\left(\Delta b_{j}\right)^{2}=\left(b_{j}-\bar{b}\right)^{2}=\left(b_{j}^{2}-2 \bar{b} b_{j}+(\bar{b})^{2}\right) \\
\overline{(\Delta b)^{2}}=\left(\overline{(b)^{2}}-2 \overline{b b}+(\bar{b})^{2}\right)=\overline{(b)^{2}}-(\bar{b})^{2}
\end{gathered}
$$

Pour chaque élément, on définit

- sa section efficace totale $\sigma=4 \pi \overline{(b)^{2}}$

- sa section efficace cohérente $\sigma_{c}=4 \pi(\bar{b})^{2}$

et on a bien :

- sa section efficace incohérente $\sigma_{i}=4 \pi \overline{(b)^{2}}-4 \pi(\bar{b})^{2}$

$$
\sigma=\sigma_{c}+\sigma_{\mathrm{i}}
$$

Dans le tableau II sont tabulées, pour chaque isotope et pour chaque élément, les longueurs de Fermi moyennes $\bar{b}$ et les sections efficaces totales $\sigma=4 \pi \overline{(b)^{2}}$. On peut en déduire la section efficace cohérente $\sigma_{c}=4 \pi(\bar{b})^{2}$ et la section efficace incohérente $\sigma_{i}=\sigma-\sigma_{c}$.

Pour les systèmes monoatomiques, on peut donc remplacer dans l'expression des sections efficaces différentielles partielles $(\bar{b})^{2}$ par $\frac{\sigma_{c}}{4 \pi}$ et $\overline{(\Delta b)^{2}}$ par $\frac{\sigma_{i}}{4 \pi}$. On a alors :

$$
\left(\frac{d^{2} \sigma}{d \Omega d E^{\prime}}\right)=\left(\frac{d^{2} \sigma}{d \Omega d E^{\prime}}\right)_{c o h}+\left(\frac{d^{2} \sigma}{d \Omega d E^{\prime}}\right)_{i n c}
$$


avec

$$
\begin{aligned}
& \left(\frac{\mathrm{d}^{2} \sigma}{\mathrm{d} \Omega \mathrm{dE} E^{\prime}}\right)_{c o h}=\frac{1}{2 \pi \hbar} \frac{\mathrm{k}^{\prime}}{\mathrm{k}} \frac{\sigma_{c}}{4 \pi} \sum_{\mathrm{ij}} \int_{-\infty}^{+\infty}\left\langle\mathrm{e}^{-\mathrm{i} \overrightarrow{\mathrm{Q}}_{\mathrm{j}}(0)} \mathrm{e}^{\mathrm{i} \overrightarrow{\mathrm{Q}} \overrightarrow{\mathrm{R}}_{\mathrm{j}}(1)}\right\rangle \mathrm{e}^{-\mathrm{i} \omega t} \mathrm{dt} \\
& \left(\frac{\mathrm{d}^{2} \sigma}{\mathrm{d} \Omega \mathrm{dE} \mathrm{E}^{\prime}}\right)_{\mathrm{inc}}=\frac{1}{2 \pi \hbar} \frac{\mathrm{k}^{\prime}}{\mathrm{k}} \frac{\sigma_{\mathrm{i}}}{4 \pi} \sum_{\mathrm{j}} \int_{-\infty}^{+\infty}\left\langle\mathrm{e}^{-\mathrm{i} \overline{\mathrm{Q}} \overline{\mathrm{R}}_{\mathrm{j}}(0)} \mathrm{e}^{\mathrm{i} \overrightarrow{\mathrm{Q}}_{\mathrm{j}}(\mathrm{t})}\right\rangle \mathrm{e}^{-\mathrm{i} \omega t} \mathrm{dt}
\end{aligned}
$$

\section{II-3 Fonctions de paires, fonctions intermédiaires et facteurs de structure dynamiques}

\section{3. 1. Fonctions de corrélation de paires}

Nous avions défini un opérateur "densité nucléaire" :

$$
\hat{\rho}(\overrightarrow{\mathrm{r}}, \mathrm{t})=\sum_{\mathbf{j}} \mathrm{b}_{\mathbf{j}} \delta\left(\overrightarrow{\mathrm{r}}-\overrightarrow{\mathrm{R}}_{\mathrm{j}}(\mathrm{t})\right)
$$

En supposant, pour simplifier, que le système est monoatomique, on utilise l'opérateur "densité de particules" :

$$
\rho(\overrightarrow{\mathrm{r}}, \mathrm{t})=\sum_{\mathrm{j}} \delta\left(\overrightarrow{\mathrm{r}}-\overrightarrow{\mathrm{R}}_{\mathrm{j}}(\mathrm{t})\right)
$$

La fonction de corrélation de paires dépendant du temps est :

$$
\begin{aligned}
\mathrm{G}(\overrightarrow{\mathrm{r}}, \mathrm{t}) & =\frac{1}{N} \int\left\langle\rho\left(\overrightarrow{\mathrm{r}}^{\prime}, 0\right) \rho\left(\overrightarrow{\mathrm{r}}^{\prime}+\overrightarrow{\mathrm{r}}, \mathrm{t}\right)\right\rangle \mathrm{d} \overrightarrow{\mathrm{r}}^{\prime} \\
& =\frac{1}{\mathrm{~N}} \sum_{\mathrm{j}^{\prime}} \int\left\langle\delta\left[\overrightarrow{\mathrm{r}}^{\prime}-\overrightarrow{\mathrm{R}}_{\mathrm{j}}(0)\right] \delta\left[\overrightarrow{\mathrm{r}}^{\prime}+\overrightarrow{\mathrm{r}}-\overrightarrow{\mathrm{R}}_{\mathrm{j}^{\prime}}(\mathrm{t})\right]\right\rangle \mathrm{d} \overrightarrow{\mathrm{r}}^{\prime}
\end{aligned}
$$

Cette fonction de corrélation de paires dépendant du temps $G(\vec{r}, t)$ correspond à la probabilité de trouver une particule en $(\vec{r}, t)$ sachant qu'il y en avait une en $(\overrightarrow{0}, 0)$ et que nous avons désignée par $\mathrm{P}[\rho(\overrightarrow{\mathrm{r}}, \mathrm{t}) \mid \rho(0,0)]$ dans le paragraphe du corréloscope.

De la même façon, la fonction d'autocorrélation de paires (self) dépendant du temps est :

$$
\mathrm{G}_{\mathrm{s}}(\overrightarrow{\mathrm{r}}, \mathrm{t})=\frac{1}{\mathrm{~N}} \sum_{\mathrm{j}} \int\left\langle\delta\left[\overrightarrow{\mathrm{r}}^{\prime}-\overrightarrow{\mathrm{R}}_{\mathrm{j}}(0)\right] \delta\left[\overrightarrow{\mathrm{r}}^{\prime}+\overrightarrow{\mathrm{r}}-\overrightarrow{\mathrm{R}}_{\mathrm{j}}(\mathrm{t})\right]\right\rangle \mathrm{d}^{\prime}
$$

qui correspond, dans l'image du corréloscope à mouches, à la probabilité de trouver en $(\vec{r}, t)$ la même mouche qu'on avait trouvée en $(\overrightarrow{0}, 0)$.

Les sections efficaces différentielles partielles, cohérentes et incohérentes, sont, à un coefficient près, les doubles transformées de Fourier de $G(\vec{r}, t)$ et $G_{s}(\vec{r}, t)$.

$\mathrm{G}(\overrightarrow{\mathrm{r}}, \mathrm{t})$ et $\mathrm{G}_{\mathrm{s}}(\overrightarrow{\mathrm{r}}, \mathrm{t})$ ont la dimension d'un [volume] ${ }^{-1}$.

\section{3. 2. Fonctions intermédiaires}

On est parfois amené à utiliser des transformées de Fourier des fonctions $G(\vec{r}, t)$ et $\mathrm{G}_{\mathrm{s}}(\overrightarrow{\mathrm{r}}, \mathrm{t})$, qui ne sont transformées de Fourier que par rapport à la variable d'espace $\overrightarrow{\mathrm{r}}$. 
On définit ainsi :

La fonction intermédiaire $I(\vec{Q}, t)$ :

$$
\begin{aligned}
\mathbf{I}(\overrightarrow{\mathrm{Q}}, \mathrm{t}) & =\int \mathrm{G}(\overrightarrow{\mathrm{r}}, \mathrm{t}) \mathrm{e}^{\mathrm{i} \overrightarrow{\mathrm{Q}}^{\mathrm{Q}} \overrightarrow{\mathrm{r}}} \mathrm{d} \overrightarrow{\mathbf{r}} \\
& =\frac{1}{\mathrm{~N}} \sum_{\mathrm{j} j^{\prime}}\left\langle\mathrm{e}^{-\mathrm{i} \overrightarrow{\mathrm{Q}} \cdot \overrightarrow{\mathrm{R}}_{\mathrm{j}}(0)} \mathrm{e}^{\mathrm{i} \overline{\mathrm{Q}} \cdot \overrightarrow{\mathrm{R}}_{j}(\mathrm{t})}\right\rangle
\end{aligned}
$$

et la fonction intermédiaire self $I_{S}(\vec{Q}, t)$ :

$$
\begin{aligned}
I_{S}(\vec{Q}, t) & =\int G_{S}(\vec{r}, t) e^{i \cdot \hat{Q} \cdot \vec{r}} d \vec{r} \\
& =\frac{1}{N} \sum_{j}\left\langle e^{-i \bar{Q} \cdot \vec{R}_{j}(0)} e^{i \bar{Q}^{-} \cdot \overline{\mathbf{R}}_{j}(t)}\right\rangle
\end{aligned}
$$

$\mathrm{I}(\overrightarrow{\mathrm{Q}}, \mathrm{t})$ et $\mathrm{I}_{s}(\overrightarrow{\mathrm{Q}}, \mathrm{t})$ sont sans dimension.

\section{3. 3. Facteurs de structure dynamiques}

Pour arriver aux sections efficaces différentielles partielles, il reste à transformer de Fourier $\mathrm{I}(\overrightarrow{\mathrm{Q}}, \mathrm{t})$ et $\mathrm{I}_{\mathrm{s}}(\overrightarrow{\mathrm{Q}}, \mathrm{t})$ par rapport au temps. On définit alors le facteur de structure dynamique $\mathrm{S}(\overrightarrow{\mathrm{Q}}, \omega){ }^{5}$ :

$$
\begin{aligned}
\mathrm{S}(\overrightarrow{\mathrm{Q}}, \omega) & =\frac{1}{2 \pi \hbar} \int_{-\infty}^{\infty} \mathrm{I}(\overrightarrow{\mathrm{Q}}, \mathrm{t}) \mathrm{e}^{-\mathrm{i} \omega t} \mathrm{dt} \\
& =\frac{1}{2 \pi \hbar \mathrm{N}} \int_{-\infty}^{\infty} \sum_{\mathrm{j} j}^{\infty}\left\langle\mathrm{e}^{-\mathrm{i} \overline{\mathrm{Q}}^{\prime} \cdot \overrightarrow{\mathrm{R}}_{\mathrm{j}}(0)} \mathrm{e}^{\mathrm{i} \overrightarrow{\mathrm{Q}} \cdot \overrightarrow{\mathrm{R}}_{f^{\prime}}(t)}\right\rangle \mathrm{e}^{-\mathrm{i} \omega t} \mathrm{dt}
\end{aligned}
$$

et le facteur de structure dynamique incohérent $S_{\mathrm{inc}}(\overrightarrow{\mathrm{Q}}, \omega)$ :

$$
\begin{aligned}
\mathrm{S}_{\mathrm{inc}}(\overrightarrow{\mathrm{Q}}, \omega) & =\frac{1}{2 \pi \hbar} \int_{-\infty}^{\infty} \mathrm{I}_{\mathrm{s}}(\overrightarrow{\mathrm{Q}}, \mathrm{t}) \mathrm{e}^{-\mathrm{i} \omega t} \mathrm{dt} \\
& =\frac{1}{2 \pi \hbar \mathrm{N}} \int_{-\infty}^{\infty} \sum_{\mathrm{j}}^{\infty}\left\langle\mathrm{e}^{-\mathrm{i} \overline{\mathrm{Q}} \cdot \overline{\mathrm{R}}_{j}(0)} \mathrm{e}^{\mathrm{i} \hat{\mathrm{Q}} \cdot \overrightarrow{\mathrm{R}}_{\mathrm{j}}(\mathrm{t})}\right\rangle \mathrm{e}^{-\mathrm{i} \omega t} \mathrm{dt}
\end{aligned}
$$

$\mathrm{S}(\overrightarrow{\mathrm{Q}}, \omega)$ et $\mathrm{S}_{\mathrm{inc}}(\overrightarrow{\mathrm{Q}}, \omega)$ ont comme dimension une [énergie] ${ }^{-1}$.

\section{3. 4. Sections efficaces différentielles partielles}

Les sections efficaces peuvent donc s'écrire (pour un système monoatomique) :

et

$$
\left(\frac{\mathrm{d}^{2} \sigma}{\mathrm{d} \Omega \mathrm{dE} \mathrm{E}^{\prime}}\right)_{\mathrm{coh}}=\frac{\mathrm{k}^{\prime}}{\mathrm{k}} \frac{\sigma_{\mathrm{c}}}{4 \pi} \mathrm{NS}(\overrightarrow{\mathrm{Q}}, \omega)
$$

\footnotetext{
$5^{5}$ Le facteur de structure dynamique est aussi appelé "scattering law", sans qu'il n'y ait d'explication logique pour ce terme
} 


$$
\left(\frac{\mathrm{d}^{2} \sigma}{\mathrm{d} \Omega \mathrm{dE} E^{\prime}}\right)_{\mathrm{inc}}=\frac{\mathrm{k}^{\prime}}{\mathrm{k}} \frac{\sigma_{\mathrm{i}}}{4 \pi} \mathrm{NS}_{\mathrm{inc}}(\overrightarrow{\mathrm{Q}}, \omega)
$$

Dans ces deux expressions, la première partie dépend des particularités de l'expérience et des neutrons, tandis que la deuxième partie est caractéristique du système étudié et représente sa fonction de réponse.

\section{II-4 Gain et perte d'énergie. Le principe de la balance détaillée.}

Par convention, $\hbar \omega$ est positif lorsque le neutron cède de l'énergie à l'échantillon (le système) : le neutron perd de l'énergie et le système en gagne lorsqu'il passe de $\lambda$ à $\lambda^{\prime}$.

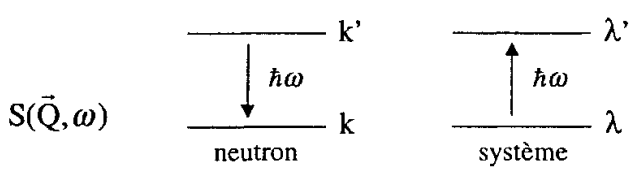

II peut se produire le processus de diffusion inverse où le système passe de $\lambda$ ' à $\lambda$ en perdant de l'énergie tandis que le neutron gagne cette énergie :

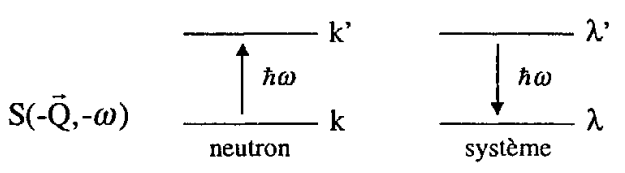

Pour les deux processus, les facteurs de structure dynamiques s'écriront exactement de la même façon, sauf la probabilité de trouver le système en $\lambda$ ou $\lambda^{\prime}$ ( $p_{\lambda}$ ou $p_{\lambda^{\prime}}$ qui interviennent dans la moyenne thermique $<\ldots>$ ).

donc :

$$
\mathrm{p}_{\lambda^{\prime}}=\mathrm{e}^{-\frac{\hbar \omega}{\mathrm{k}_{\mathrm{B}} \mathrm{T}}} \mathrm{p}_{\lambda}
$$

$$
\mathrm{S}(-\overrightarrow{\mathrm{Q}},-\omega)=\mathrm{e}^{-\frac{\hbar \omega}{\mathbf{k}_{\mathrm{B}} \mathrm{T}}} \mathrm{S}(\overrightarrow{\mathrm{Q}}, \omega)
$$

Si le système est centro-symétrique, on a alors $S(-\vec{Q}, \omega)=S(\vec{Q}, \omega)$, et on peut écrire:

$$
\mathrm{S}(\overrightarrow{\mathrm{Q}},-\omega)=\mathrm{e}^{-\frac{\hbar \omega}{\mathrm{k}_{\mathrm{B}} \mathrm{T}}} \mathrm{S}(\overrightarrow{\mathrm{Q}}, \omega)
$$

C'est le principe de la balance détaillée. Il indique que si on travaille en perte d'énergie pour le neutron ( $\hbar \omega>0)$, l'intensité diffusée sera plus forte car, entre les deux états $\lambda$ et $\lambda^{\prime}$, la probabilité est plus forte de trouver le système dans l'état de plus basse énergie.

\section{II-5 Diffusion élastique et diffusion inélastique}

Pour tous les systèmes, on peut décomposer la fonction de corrélation de paire dépendant du temps de la façon suivante : 


$$
\begin{aligned}
G(\vec{r}, t) & =G(\vec{r}, \infty)+G^{\prime}(\vec{r}, t) \\
& \text { avec } \lim _{t \rightarrow \infty} G^{\prime}(\vec{r}, t)=0
\end{aligned}
$$

De même, on peut décomposer la fonction intermédiaire :

$$
\begin{aligned}
I(\vec{Q}, t) & =I(\vec{Q}, \infty)+I^{\prime}(\vec{Q}, t) \\
& \operatorname{avec} \lim _{t \rightarrow \infty} I^{\prime}(\vec{Q}, t)=0
\end{aligned}
$$

Ce qui nous donne pour le facteur de structure dynamique :

$$
\begin{aligned}
\mathrm{S}(\overrightarrow{\mathrm{Q}}, \omega) & =\frac{1}{2 \pi \hbar} \int_{-\infty}^{\infty}\left[\mathrm{I}(\overrightarrow{\mathrm{Q}}, \infty)+\mathrm{I}^{\prime}(\overrightarrow{\mathrm{Q}}, \mathrm{t})\right] \mathrm{e}^{-\mathrm{i} \omega t} \mathrm{dt} \\
& =\frac{\delta(\omega)}{\hbar} \mathrm{I}(\overrightarrow{\mathrm{Q}}, \infty)+\frac{1}{2 \pi \hbar} \int_{-\infty}^{\infty} \mathrm{I}^{\prime}(\overrightarrow{\mathrm{Q}}, \mathrm{t}) \mathrm{e}^{-\mathrm{i} \omega t} \mathrm{dt} \\
& =\mathrm{S}^{\mathrm{el}}(\overrightarrow{\mathrm{Q}}, \omega)+\mathrm{S}^{\text {inel }}(\overrightarrow{\mathrm{Q}}, \omega)
\end{aligned}
$$

Ce qui donne pour la section efficace différentielle partielle :

avec

$$
\left(\frac{d^{2} \sigma}{d \Omega d E^{\prime}}\right)_{c o h}=\left(\frac{d^{2} \sigma}{d \Omega d E^{\prime}}\right)_{c o h}^{\text {el }}+\left(\frac{d^{2} \sigma}{d \Omega d E^{\prime}}\right)_{c o h}^{\text {inel }}
$$

$$
\left(\frac{\mathrm{d}^{2} \sigma}{\mathrm{d} \Omega \mathrm{dE}^{\prime}}\right)_{\mathrm{coh}}^{\mathrm{el}}=\frac{\mathrm{k}^{\prime}}{\mathrm{k}} \frac{\sigma_{\mathrm{c}}}{4 \pi} \frac{\mathrm{N}}{\hbar} \delta(\omega) \mathrm{I}(\overrightarrow{\mathrm{Q}}, \infty)
$$

en notant que $\mathbf{k}^{\prime} \mathbf{k}^{\prime}$ et après intégration sur $\mathbf{E}^{\prime}=\hbar \omega$ :

$$
\left(\frac{\mathrm{d} \sigma}{\mathrm{d} \Omega}\right)_{\mathrm{coh}}^{\mathrm{cl}}=\int\left(\frac{\mathrm{d}^{2} \sigma}{\mathrm{d} \Omega \mathrm{dE}}\right)_{\mathrm{coh}}^{\mathrm{el}} \mathrm{dE}^{\prime}=\frac{\sigma_{\mathrm{c}}}{4 \pi} \mathrm{N} \mathrm{I}(\overrightarrow{\mathrm{Q}}, \infty)
$$

avec

$$
\mathrm{I}(\overrightarrow{\mathrm{Q}}, \infty)=\frac{1}{\mathrm{~N}} \sum_{\mathrm{ji}}\left\langle\mathrm{e}^{-\mathrm{i} \overrightarrow{\mathrm{Q}} \cdot \overrightarrow{\mathrm{R}}_{\mathrm{j}}(0)} \mathrm{e}^{\mathrm{i} \overrightarrow{\mathrm{Q}} \cdot \overrightarrow{\mathrm{R}}_{\mathrm{f}}(\infty)}\right\rangle
$$

et

$$
\left(\frac{\mathrm{d} \sigma}{\mathrm{d} \Omega}\right)_{\mathrm{coh}}^{\mathrm{el}}=\frac{\sigma_{\mathrm{c}}}{4 \pi} \sum_{\mathrm{ij}}\left\langle\mathrm{e}^{-\mathrm{i} \mathrm{Q} \cdot \overrightarrow{\mathrm{R}}_{\mathrm{j}}(0)} \mathrm{e}^{\mathrm{i} \overrightarrow{\mathrm{Q}}^{\cdot \overrightarrow{\mathrm{R}}_{f}(\omega)}}\right\rangle
$$

Par exemple, pour un cristal rigide, formé d'atomes identiques et fixes, aux noeuds d'un réseau de Bravais

$$
\rho(\overrightarrow{\mathrm{r}})=\sum_{\vec{l}} \delta(\overrightarrow{\mathrm{r}}-\vec{l})
$$

on a comme section efficace différentielle cohérente : 


$$
\left(\frac{\mathrm{d} \sigma}{\mathrm{d} \Omega}\right)_{\mathrm{coh}}^{\mathrm{el}}=\frac{\sigma_{\mathrm{c}}}{4 \pi}\left|\sum_{i} \mathrm{e}^{\mathrm{i} \mathrm{Q} \cdot \hat{i}}\right|^{2}
$$

C'est là un résultat bien connu: la somme $\sum_{i} \mathrm{e}^{\mathrm{i} \bar{Q} \cdot \hat{l}}$ vaut en général zéro, sauf si le vecteur $\vec{Q}$ se trouve coincider avec un noeud du réseau réciproque. En dehors de ces noeuds, la section efficace cohérente est nulle.

En effet:

$$
\left|\sum_{\vec{i}} \mathrm{e}^{\mathrm{i} \cdot \bar{i} \cdot \vec{i}}\right|^{2}=\frac{\mathrm{N}(2 \pi)^{3}}{\mathrm{v}_{0}} \sum_{\vec{i}} \delta(\overrightarrow{\mathrm{Q}}-\vec{\tau})
$$

où Nreprésente le nombre de mailles (ici d'atomes), $\mathrm{v}_{0}$ le volume d'une maille du réseau direct et où les $\vec{\tau}$ correspondent aux différents noeuds du réseau réciproque ${ }^{6}$. On a alors la loi de Bragg pour un cristal monoatomique rigide :

$$
\left(\frac{\mathrm{d} \sigma}{\mathrm{d} \Omega}\right)_{\mathrm{coh}}^{\mathrm{el}}=\frac{\mathrm{N}(2 \pi)^{3}}{\mathrm{v}_{0}}(\overline{\mathrm{b}})^{2} \sum_{\vec{z}} \delta(\overrightarrow{\mathrm{Q}}-\vec{\tau})
$$

Cette loi se généralise pour un cristal formé d'atomes non identiques qui vibrent autour de leur position d'équilibre :

$$
\left(\frac{\mathrm{d} \sigma}{\mathrm{d} \Omega}\right)_{\text {coh }}^{\mathrm{cl}}=\frac{\mathrm{N}(2 \pi)^{3}}{\mathrm{v}_{0}}\left|\sum_{\mathrm{j}} \widetilde{\mathrm{b}}_{\mathrm{j}} \mathrm{e}^{\mathrm{i} \overline{\mathrm{Q}} \overrightarrow{\mathrm{R}}_{j}} \mathrm{e}^{-\mathrm{w}_{j}}\right|^{2} \sum_{\vec{i}} \delta(\overrightarrow{\mathrm{Q}}-\vec{\tau})
$$

avec un facteur de structure nucléaire

$$
\mathrm{F}_{\mathrm{N}}(\overrightarrow{\mathrm{Q}})=\sum_{\mathrm{j}} \overline{\mathrm{b}}_{\mathrm{j}} \mathrm{e}^{\mathrm{i} \overrightarrow{\mathrm{Q}} \cdot \overrightarrow{\mathrm{K}}_{j}} \mathrm{e}^{-\mathrm{W}_{\mathrm{j}}}
$$

qui porte sur tous les atomes $\mathrm{j}$ d'une maille et qui inclut le facteur de Debye-Waller $\mathrm{e}^{-\mathrm{w}_{j}}$ de chaque atome.

On a un traitement équivalent pour la diffusion incohérente:

$$
\begin{aligned}
& \mathrm{G}_{\mathrm{s}}(\overrightarrow{\mathrm{r}}, \mathrm{t})=\mathrm{G}_{\mathrm{s}}(\overrightarrow{\mathrm{r}}, \infty)+\mathrm{G}_{\mathrm{s}}^{\prime}(\overrightarrow{\mathrm{r}}, \mathrm{t}) \\
& \text { avec } \lim _{t \rightarrow \infty} G_{s}^{\prime}(\vec{r}, t)=0 \\
& I_{s}(\vec{Q}, t)=I_{s}(\vec{Q}, \infty)+I_{s}^{\prime}(\vec{Q}, t) \\
& \text { avec } \lim _{t \rightarrow \infty} I_{S}^{\prime}(\vec{Q}, t)=0
\end{aligned}
$$

\footnotetext{
${ }^{6}$ On rappelle ici que les 3 vecteurs de base du réseau réciproque sont définis par $\vec{\tau}_{1}=\frac{2 \pi}{v_{0}} \vec{a}_{2} \wedge \vec{a}_{3}, \quad \vec{\tau}_{2}=\frac{2 \pi}{v_{0}} \vec{a}_{3} \wedge \vec{a}_{1}, \quad \vec{\tau}_{3}=\frac{2 \pi}{v_{0}} \vec{a}_{1} \wedge \vec{a}_{2}$
} 
et

$$
\begin{aligned}
S_{\text {inc }}(\overrightarrow{\mathrm{Q}}, \omega) & =\frac{1}{2 \pi \hbar} \int_{-\infty}^{\infty}\left[\mathrm{I}_{s}(\overrightarrow{\mathrm{Q}}, \infty)+\mathrm{I}_{\mathrm{s}}(\overrightarrow{\mathrm{Q}}, \mathrm{t})\right] \mathrm{e}^{-\mathrm{i} \omega t} \mathrm{dt} \\
= & \frac{\delta(\omega)}{\hbar} \mathrm{I}_{S}(\overrightarrow{\mathrm{Q}}, \infty)+\frac{1}{2 \pi \hbar} \int_{-\infty}^{\infty} \mathrm{I}_{S}^{\prime}(\overrightarrow{\mathrm{Q}}, \mathrm{t}) \mathrm{e}^{-\mathrm{i} \omega t} \mathrm{dt} \\
= & \mathrm{S}_{\mathrm{inc}}^{\mathrm{el}}(\overrightarrow{\mathrm{Q}}, \omega)+\mathrm{S}_{\mathrm{inc}}^{\mathrm{inel}}(\overrightarrow{\mathrm{Q}}, \omega)
\end{aligned}
$$

$$
\left(\frac{d^{2} \sigma}{d \Omega d E^{\prime}}\right)_{i n c}=\left(\frac{d^{2} \sigma}{d \Omega d E^{\prime}}\right)_{i n c}^{e l}+\left(\frac{d^{2} \sigma}{d \Omega d E^{\prime}}\right)_{i n c}^{i n e l}
$$

qui, pour la partie élastique, après intégration sur $E^{\prime}$ donne :

$$
\left(\frac{\mathrm{d} \sigma}{\mathrm{d} \Omega}\right)_{\mathrm{inc}}^{\mathrm{el}}=\frac{\sigma_{\mathrm{i}}}{4 \pi} \sum_{\mathrm{j}}\left\langle\mathrm{e}^{-\mathrm{i} \overrightarrow{\mathrm{Q}} \cdot \overrightarrow{\mathrm{R}}_{\mathrm{j}}(0)} \mathrm{e}^{\mathrm{i} \overrightarrow{\mathrm{Q}}_{\mathrm{R}} \cdot \overrightarrow{\mathrm{R}}_{\mathrm{j}}(\infty)}\right\rangle
$$

Dans le cas du cristal formé d'atomes identiques, fixes, aux noeuds du réseau de Bravais,

$$
\left(\frac{\mathrm{d} \sigma}{\mathrm{d} \Omega}\right)_{\mathrm{inc}}^{\mathrm{el}}=\frac{\sigma_{\mathrm{i}}}{4 \pi} \sum_{\vec{i}} \mathrm{e}^{-\mathrm{i} \overrightarrow{\mathrm{Q}} \cdot \vec{l}} \mathrm{e}^{\mathrm{i} \hat{\mathrm{Q}} \cdot \vec{l}}=\frac{\mathrm{N} \sigma_{\mathrm{i}}}{4 \pi}
$$

La diffusion incohérente, dans ce cas est isotrope car elle est indépendante de $\vec{Q}$.

Mais si on considère un cristal où les atomes vibrent autour de leur position d'équilibre, on retrouve le facteur de Debye-Waller qui représente la corrélation qui existe entre la position de l'atome à un moment donné et la position de ce même atome beaucoup plus tard:

$$
\left(\frac{\mathrm{d} \sigma}{\mathrm{d} \Omega}\right)_{\mathrm{inc}}^{\mathrm{el}}=\sum_{\mathrm{j}} \frac{\left(\sigma_{\mathrm{i}}\right)_{\mathrm{j}}}{4 \pi} \mathrm{e}^{-\mathrm{i} \mathrm{Q} \cdot \overline{\mathrm{k}}_{\mathrm{j}}(0)} \mathrm{e}^{\mathrm{i} \overline{\mathrm{Q}}^{-\overline{\mathrm{j}}_{\mathrm{j}}(\infty)}}=\mathrm{N} \frac{\sigma_{\mathrm{i}}}{4 \pi} \mathrm{e}^{-2 \mathrm{~W}}
$$

\section{II-6 Les instruments de diffusion neutronique}

\section{6. 1. Les diffractomètres (diffusion élastique)}

Pour l'étude des structures, on n'est intéressé que par la fonction $S(\vec{Q})$. Les instruments utilisés négligent la dépendence en $\omega$ et considèrent que toute la diffusion est élastique. La diffusion élastique par la matière cristallisée est le plus souvent appelée "diffraction" et les appareils correspondants sont les "diffractomètres".

Une première série d'instruments implique un faisceau incident monochromatique. Ce faisceau est obtenu par réflexion de Bragg sur une série de plans réflecteurs (hkl) d'un gros cristal appelé monochromateur :

$$
2 d_{h k l} \sin \theta=\lambda
$$


Ce monochromateur sélectionne une longueur d'onde unique (un seul $\vec{k}$ ) pour le faisceau tombant sur l'échantillon. Un détecteur mesure l'intensité (section efficace différentielle) diffusée dans la direction $\overrightarrow{\mathrm{k}}^{\prime}$ où il se trouve. Le détecteur bouge et explore les différents $\overrightarrow{\mathrm{k}}^{\prime}$ (figure 10).

Figure 10: diffractomètre à faisceau monochromatique

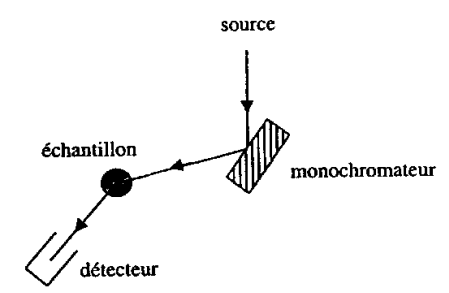

En diffraction classique, on distingue -les diffractomètres à poudres -les diffractomètres à monocristaux

-avec cercle d'Euler (4 cercles) -à bras levant

Pour la diffusion neutronique à petits angles (DNPA), on utilise une grande longueur d'onde et le monochromateur est remplacé par un sélecteur de vitesse mécanique (figure 11).

Figure 11: diffractomètre de diffusion à petits angles

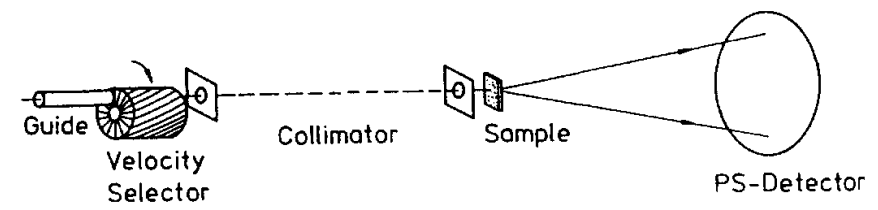

Une deuxième série d'instruments est basée sur la mesure du temps de vol. Dans ce type d'instruments, les neutrons arrivent par bouffées, soit que la source est pulsée, soit que le faisceau primaire est haché par un chopper. Là encore, on considère que toute la diffusion est élastique et, pour explorer les différents vecteurs de diffusion $\vec{Q}$, on fixe le détecteur (angle fixe) et on mesure les différentes longueurs d'onde qui sont diffusées (figure 12). En effet, pour chaque neutron détecté, on enregistre le temps $\tau$ qu'il a mis pour aller du chopper jusqu'au compteur, et sa vitesse est donnée par:

$$
\mathrm{v}=\frac{\tau}{\mathrm{L}_{1}+\mathrm{L}_{2}}
$$

Figure 12: diffractomètre à temps de vol

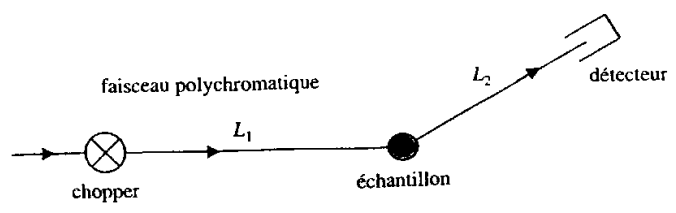




\section{6. 2. Les spectromètres (diffusion inélastique)}

Ces appareils permettent de connaître l'énergie (ou le vecteur d'onde) des neutrons avant et après le processus de diffusion.

\section{Spectromètre à triple axe}

C'est un appareil qui s'obtient en ajoutant, après l'échantillon, un troisième axe à un diffractomètre à faisceau monochromatique. Ce troisième axe porte un cristal analyseur qui permet, par réflexion de Bragg, de mesurer l'énergie des neutrons diffusés par l'échantillon (figure 13)

Figure 13: Spectromètre à triple axe

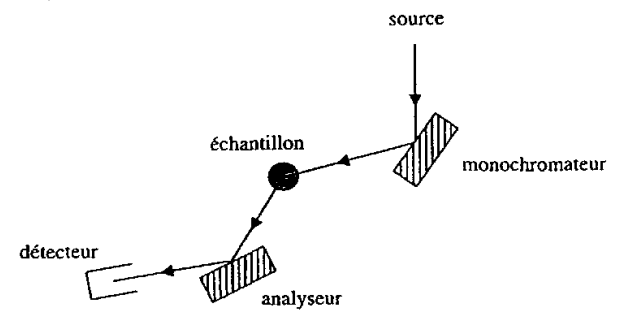

\section{Spectromètre à temps de vol (ou time of flight)}

II existe plusieurs façons d'utiliser le temps de vol et de le combiner à la diffraction de Bragg sur un cristal pour analyser les énergies avant et après diffusion sur l'échantillon comme le montre le tableau IV.

\section{Tableau IV}

Les différents types de spectromètres à temps de vol

\begin{tabular}{|l|l|l|}
\hline avant diffusion & après diffusion & spectromètre \\
\hline temps de vol & temps de vol & spectromètre à double chopper \\
\hline cristal & temps de vol & spectromètre à cristal tournant et temps de vol \\
\hline temps de vol & cristal & spectromètre à temps de vol et géométrie inverse \\
\hline
\end{tabular}

\section{6. 3. Les instruments inélastiques à très haute résolution}

\section{Les instruments à rétrodiffusion (ou backscattering)}

Ce sont des appareils qui, comme les spectromètres à triple axe, comportent un monochromateur, un échantillon et un analyseur, mais où monochromateur et analyseur sont des cristaux parfaits fonctionnant en rétrodiffusion $\left(2 \theta=180^{\circ}\right)$.

En différenciant la loi de Bragg pour obtenir la résolution en longueur d'onde

$$
\left(\frac{\Delta \lambda}{\lambda}\right)^{2}=\left(\frac{\Delta d}{d}\right)^{2}+\left(\frac{\Delta \theta}{\operatorname{tg} \theta}\right)^{2}
$$

on voit que le deuxième terme disparait pratiquement pour $\theta=90^{\circ}$ et la résolution ne dépend plus que du premier terme, c'est à dire de la perfection du cristal (monochromateur ou analyseur). 
Les neutrons rétrodiffractés par le monochromateur reviennent sur leurs pas et, grâce à un déflecteur (un cristal à large bande passante), sont envoyés sur l'échantillon. Après diffusion par l'échantillon, les neutrons sont rétrodiffractés par l'analyseur, retraversent l'échantillon et tombent sur un détecteur. En fait il y a un grand nombre d'analyseurs et de détecteurs (figure 14). Un chopper permet d'éliminer les neutrons qui iraient directement de l'échantillon au détecteur, sans passer par l'analyseur.

Figure 14: Spectromètre à rétrodiffusion

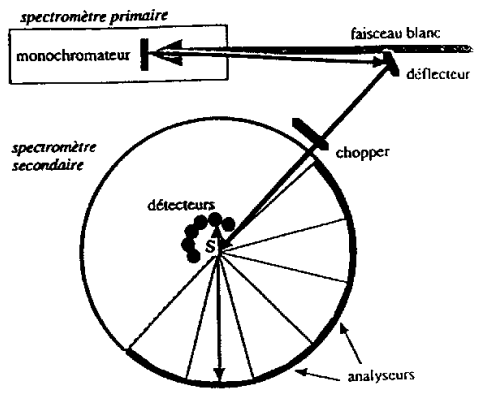

L'exploration en énergie se fait par balayage de la longueur d'onde incidente -soit par effet Doppler, en imprimant un mouvement au monochromateur -soit par chauffage du monochromateur, ce qui entraîne une variation continue du paramètre $d$.

On peut ainsi atteindre des résolutions meilleures que le $\mu \mathrm{eV}$.

\section{Les instruments à écho de spin (ou neutron spin echo)}

$\mathrm{Ce}$ sont des instruments pour lesquels la mesure du changement de vitesse du neutron avant et après le processus de diffusion se fait en étudiant le nombre de rotations de Larmor (quelques millions) que font les spins des neutrons en passant dans une zone de champ magnétique: les neutrons les plus rapides font moins de précessions, les neutrons les plus lents font plus de précessions.

Le faisceau est rendu monochromatique par un sélecteur de vitesses, polarisé par un supermiroir et les spins sont mis en rotation autour du champ $B_{0}$ dans un premier solénoïde (figure 15). Cependant, dû à la distribution en longueurs d'onde $\Delta \lambda$ (en vitesses $\Delta v$ ) du faisceau (grossièrement monochromatique seulement), la polarisation du faisceau se perd rapidement dans cette première zone de précession.

Figure 15: spectromètre à écho de spin

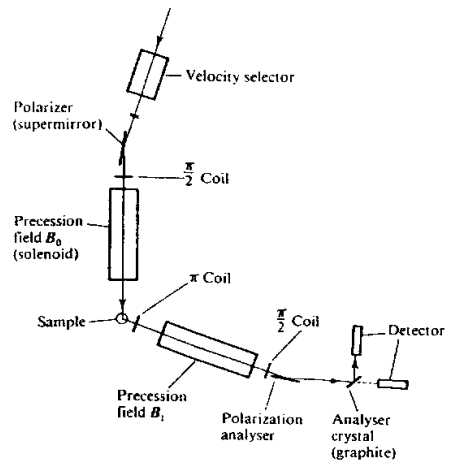


Au niveau de l'échantillon, un retournement des spins est effectué (diffusion avec spin flip ou flipper $\pi$ ). De ce fait, les spins qui avaient pris de l'avance dans leur rotation se retrouvent en retard, tandis que ceux qui avaient pris du retard se retrouvent en avance.

Après diffusion sur l'échantillon une deuxième zone de précession, identique à la première fait précesser les spins. A la sortie de cette zone, les retards et les avances de rotation se trouvant compensées, un écho de la polarisation initiale est retrouvé.

Figure 16: Variations de la polarisation en fonction du champ (scan symétrique)

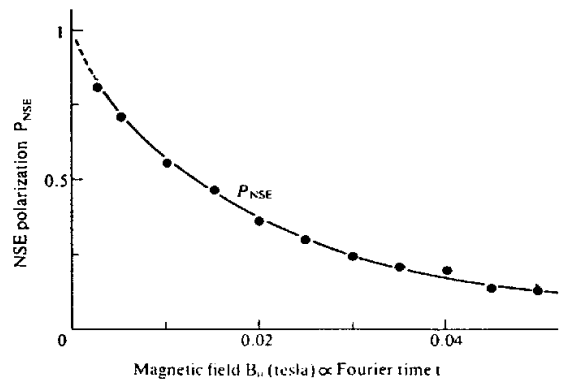

La variation de cette polarisation en fonction du champ (figure 16) dans les deux bobines (scan symétrique) représente la fonction intermédiaire $I(\vec{Q}, t)$ du système $(t$ est proportionnel à $\mathrm{B}_{0}$ ).

Avec le spin echo, des résolutions en énergie inférieures au neV peuvent être atteintes.

II. 6. 4. Les domaines d'utilisation des différents instruments de diffusion inélastique

Les domaines accessibles en transfert d'énergie et en transfert de moment (vecteur de diffusion) sont représentés sur la figure 17 pour les différents instruments de diffusion inélastique.

Figure 17: Les domaines accessibles en $Q$ et en $E$ pour les différents spectromètres. Les symboles sont les suivants : TOF pour temps de vol, BS pour rétrodiffusion et NSE pour écho de spin

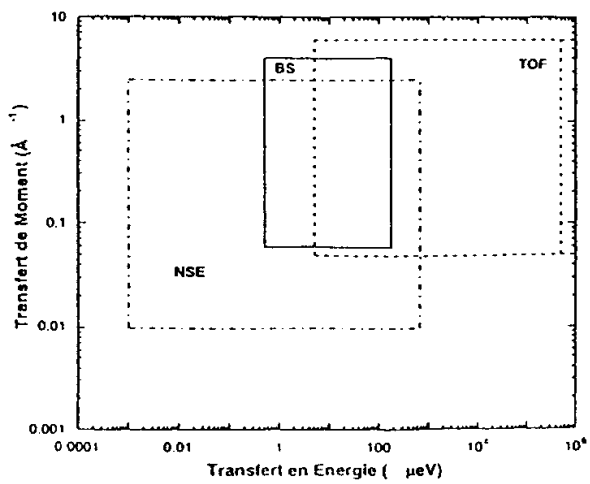




\section{Ce que I'on peut voir avec les neutrons}

Avec des neutrons, on peut se concentrer sur l'étude des structures, l'étude des dynamiques, ou encore l'étude des relations qui existent entre dynamique et structure.

\section{III-1 Voir la structure}

Etudier une structure c'est étudier comment sont situés les atomes les uns par rapport aux autres. C'est donc la diffusion cohérente qui est appropriée:

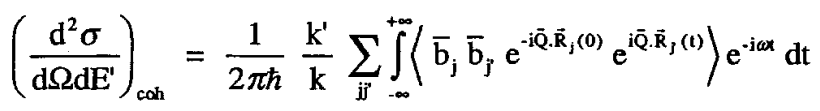

généralisation de la relation (65) pqur des systèmes composés de plusieurs types d'atomes. Le plus souvent, mais pas toujours (c'est le cas des liquides), la corrélation de structure donnée dans la formule précédente est indépendante du temps et la diffusion est élastique:

$$
\left(\frac{\mathrm{d} \sigma}{\mathrm{d} \Omega}\right)_{\mathrm{coh}}^{\mathrm{cl}}=\sum_{\mathrm{j} \mathrm{j}^{\prime}}\left\langle\overline{\mathrm{b}}_{\mathrm{j}} \overline{\mathrm{b}}_{\mathrm{j}^{\prime}} \mathrm{e}^{-\mathrm{i} \overrightarrow{\mathrm{Q}} \cdot \overrightarrow{\mathrm{k}}_{\mathrm{j}}} \mathrm{e}^{\mathrm{i} \overrightarrow{\mathrm{Q}} \cdot \overline{\mathrm{k}}_{\mathrm{j}}}\right\rangle=\left|\sum_{\mathbf{j}}\left\langle\overline{\mathrm{b}}_{\mathrm{j}} \mathrm{e}^{\mathrm{i} \overline{\mathrm{Q}} \cdot \overrightarrow{\mathrm{R}}_{j}}\right\rangle\right|^{2}
$$

généralisation des relations (86) et (87).

\section{1. 1. Structures cristallines}

Les différentes étapes de l'étude de la structure de la glace illustrent bien la puissance de l'outil neutronique.

\section{1ere étape: diffraction sur poudre}

L'étude aux rayons $X$ avait permis de localiser les positions des oxygènes, sur des tétraèdres, mais pas celle des hydrogènes. Quatre modèles étaient alors en présence (figure 18): le modèle (i) de Barnes avec l'hydrogène au milieu, entre les deux oxygènes, le modèle (ii) de Bernal et Fowler avec les deux hydrogènes de la molécule d'eau sur deux sites bien définis autour de chaque oxygène, le modèle (iii)

Figure 18: les différents

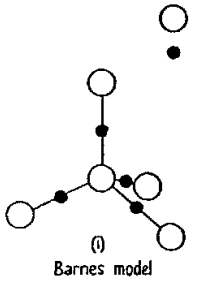

$$
\text { Oxygen }
$$

$$
\text { - Hydrogen }
$$

$$
\text { modèles proposés }
$$
pour la glace

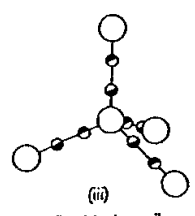

Pauling "half-hydrogen" model
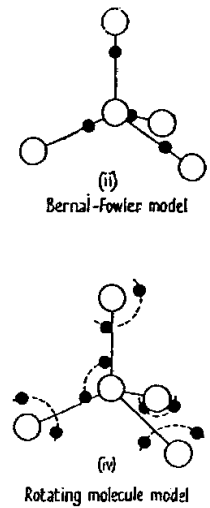
préconisé par Pauling et dit "half-hydrogen" avec une occupation aléatoire des sites possibles d'hydrogène et le modèle (iv) dit "rotating molecule" où les deux hydrogènes de la molécule tournent de façon continue autour de l'oxygène.

Une expérience de Wollan et al [6] sur de la glace deutérée polycristalline a montré que des quatre modèles, c'était le modèle (iii) qui était juste: Sur les quatre positions possibles autour de chaque oxygène, deux seulement sont occupées, et cette occupation se fait de façon aléatoire.

\section{2eme étape: diffraction sur monocristal}

Une confirmation des conclusions précédentes a été apportée par diffraction sur un monocristal de glace deutérée par Peterson et Levy [7]. La carte de Fourier obtenue à partir des intensités expérimentales (figure 19) montre bien que, entre deux atomes d'oxygène, les deux positions possibles pour l'hydrogène sont occupées par des atomes de deutérium avec un taux de remplissage de 1/2.

Figure 19: Projection de Fourier de la densité nucléaire de la glace deutérée (expérience sur monocristal)

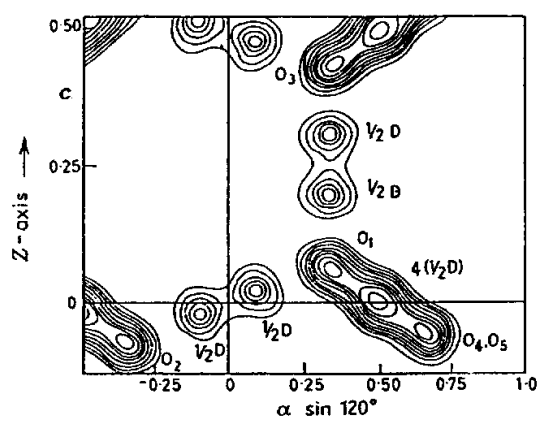

Une contradiction restait néanmoins à élucider: le modèle qui s'avérait être le modèle correct donnait une distance $\mathrm{OH}$ de $1.01 \AA$ dans la glace alors qu'elle est de $0.96 \AA$ dans la vapeur (molécule libre) et un angle $\mathrm{HOH}$ de $109.5^{\circ}$ dans la glace ('langle du tétraèdre), alors qu'il n'est que $104.5^{\circ}$ dans la molécule libre.

\section{3eme étape: diffraction à haute résolution}

L'étude précédente a été reprise en 1986 [8], avec un diffractomètre à monocristal installé sur une source chaude, qui permettait de travailler à très basse longueur d'onde $(0.50 \AA)$ et de collecter ainsi un grand nombre de réflexions. Qui dit explorer loin le réseau réciproque dit aussi obtenir des détails de haute résolution dans le réseau réel.

Figure 20: Transformée de Fourier de la densité nucléaire de la glace deutérée (expérience sur monocristal à haute résolution)

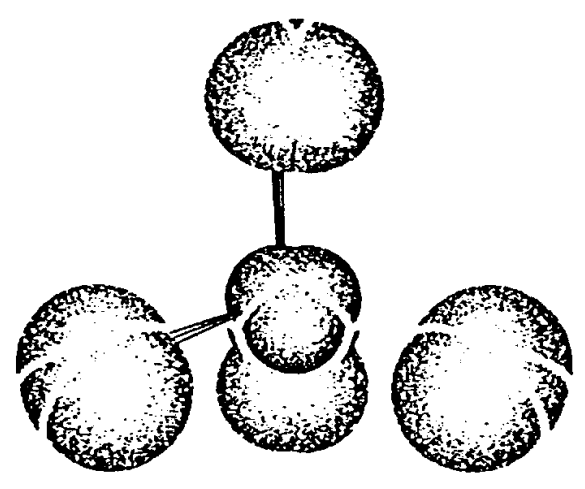


La figure obtenue par transformée de Fourier, et représentée sur la figure 20, montre que le volume représentant la probabilité de présence du deutérium n'avait pas une symétrie sphérique mais présentait 3 lobes, tandis que celui représentant l'oxygène était un volume à 6 lobes. Ainsi, pour chaque molécule $\mathrm{H}_{2} \mathrm{O}$, l'atome d'oxygène était légèrement décalé par rapport à sa position théorique au sommet du tétraèdre, tandis que les atomes d'hydrogène (de deutérium) l'étaient par rapport à leur position théorique sur la ligne $\mathrm{O}-\mathrm{O}$. La distance $\mathrm{OH}$ est alors de 0.975(5) $\AA$ et l'angle $\mathrm{HOH}$ vaut $107^{\circ}$, ce qui réconcilie le modèle de Pauling avec les connaissances que l'on avait de la molécule libre.

\section{1. 2. Structures magnétiques}

Le pendant de la formule (101) pour les structures magnétiques est:

$$
\left(\frac{\mathrm{d} \sigma}{\mathrm{d} \Omega}\right)_{\mathrm{coh}}^{\mathrm{el}}=\left|\sum_{\mathrm{j}}\left\langle\overrightarrow{\mathrm{M}}_{\perp \mathrm{j}} \mathrm{f}_{\mathrm{j}}(\mathrm{Q}) \mathrm{e}^{\mathrm{i} \overrightarrow{\mathrm{Q}} \cdot \overrightarrow{\mathrm{R}}_{\mathrm{j}}}\right\rangle\right|^{2}
$$

qui montre que la section efficace est caractéristique à la fois de la position des atomes magnétiques mais aussi de la direction des moments qu'ils portent.

La première étude de structure magnétique a été faite pas Shull et Smart en 1949 [9] sur MnO. Les spectres de diffraction sur poudre sont représentés sur la figure 21 . Le spectre à température ambiante est représentatif de l'ordre nucléaire tandis que le spectre à basse température, en dessous de la température d'ordre $\left(T_{N}=120 \mathrm{~K}\right)$, représente à la fois l'ordre nucléaire et l'ordre magnétique. L'apparition de raies de surstructure montre que la maille magnétique est deux fois plus grande que la maille chimique, confirmant le modèle imaginé par Néel 17 ans auparavant.

Figure 21: Structure antiferromagnétique de $\mathrm{MnO}$, diagrammes de poudres à $293 \mathrm{~K}$ et à $80 \mathrm{~K}$.
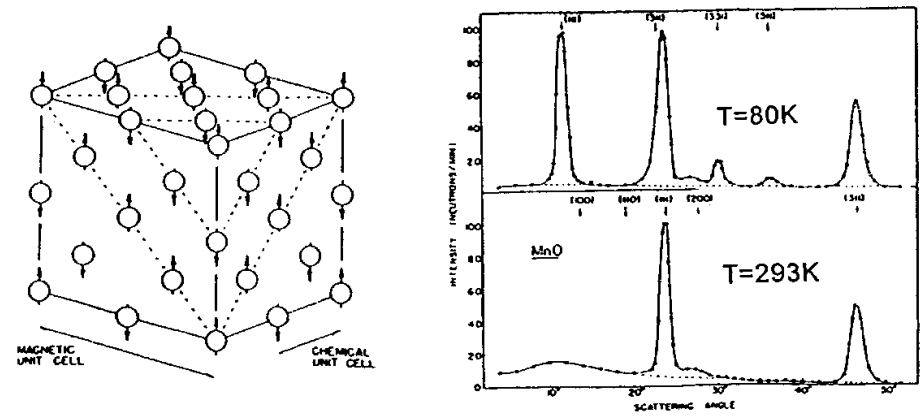

Pour cette expérience, le diagramme magnétique de $\mathrm{MnO}$ (figure 21) avait été indexé dans la maille magnétique, c'est à dire la maille cubique double de la maille nucléaire. Aujourd'hui, on décrit les structures magnétiques en définissant leur vecteur de propagation $\vec{k}$, vecteur tel que le moment magnétique $\overrightarrow{\mathrm{M}}_{1}$ dans la maille $\overrightarrow{\mathrm{l}}$ est donné par:

$$
\overrightarrow{\mathbf{M}}_{1}=\overrightarrow{\mathbf{M}} \mathrm{e}^{-\mathrm{i} \overrightarrow{\mathbf{k}} \mathbf{i}}
$$

Les réflexions magnétiques s'indexent alors de la façon suivante dans la maille nucléaire: 


$$
\overrightarrow{\mathrm{Q}}=\vec{\tau} \pm \overrightarrow{\mathrm{k}}
$$

deux satellites + et -, de part et d'autre de chaque noeud $\vec{\tau}$ du réseau réciproque de la maille nucléaire. La structure magnétique de MnO correspond à un vecteur de propagation $\overrightarrow{\mathrm{k}}=\left(\frac{1}{2}, \frac{1}{2}, \frac{1}{2}\right)$.

Ainsi, on a trouvé qu'il existait une grande variété de structures, comme par exemple la structure hélicoïdale de MnAuz [10], où le vecteur de propagation $\vec{k}=\left(0,0, \mathrm{k}_{\mathrm{z}}\right)$ est non commensurable avec le réseau réciproque. Les moments magnétiques sont tous parallèles dans un même plan perpendiculaire à l'axe $\overrightarrow{\mathrm{c}}$ et tournent tous du même angle quand on passe d'un plan au plan voisin (figure 22).
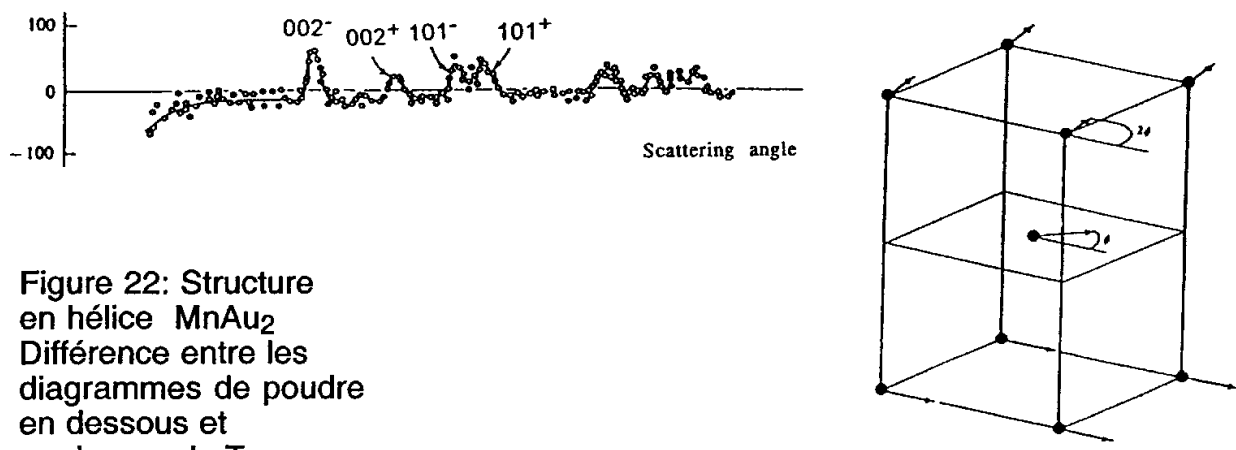

Figure 22: Structure

en hélice $\mathrm{MnAu}_{2}$

Différence entre les

diagrammes de poudre

en dessous et

en dessus de $T_{N}$

Scattering angle

\section{1. 3. Densités de spin (neutrons polarisés)}

Au delà de l'arrangement des moments magnétiques d'une structure ordonnée, il est possible d'aller plus loin dans les détails et d'obtenir par diffraction de neutrons la densité d'aimantation (densité de spin) des atomes magnétiques, c'est à dire la description du nuage magnétique électronique autour de chaque atome. Pour avoir les détails de cette distribution, il faut mesurer loin dans le réseau réciproque, donc mesurer des contributions magnétiques faibles. Pour cela, l'utilisation des neutrons polarisés est indiquée.

Figure 23: Densité d'aimantation dans un cristal de fer

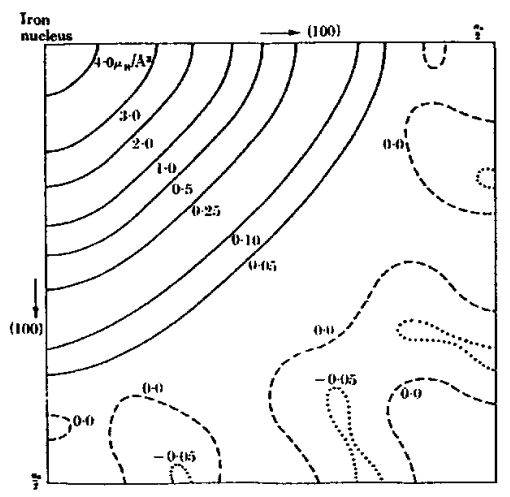


La première étude de ce type a été réalisée par Shull en 1960 [11] sur un monocristal de fer (structure cubique centrée). Une section de la densité de spin est représentée sur la figure 23. Elle montre que la densité des électrons $3 d$ est un peu asymétrique, plus forte dans les directions des arêtes du cube. Elle montre aussi que loin des noyaux, il y a des zones où l'aimantation des électrons de conduction est négative, c'est à dire opposée à l'aimantation du cristal.

\section{1. 4. Mesures de contraintes à l'intérieur de la matière}

Alors que les jauges de contraintes ne peuvent être utilisées qu'à la surface des solides, l'examen des variations relatives de distance réticulaire $\Delta \mathrm{d} / \mathrm{d}$ dans la matière apporte une connaissance dans le volume. Cette quantité se mesure avec une très bonne précision par la position de l'angle de Bragg d'une raie forte. Avec une collimation suffisamment fine, pour le faisceau incident comme pour le faisceau diffracté, on sonde un volume de l'ordre du $\mathrm{mm}^{3}$. Enfin, en déplaçant l'échantillon par un système de translations, on peut réaliser une cartographie point par point de la contrainte à l'intérieur d'un solide.

Figure 24: Variations des contraintes à l'intérieur d'un rail de chemin de fer

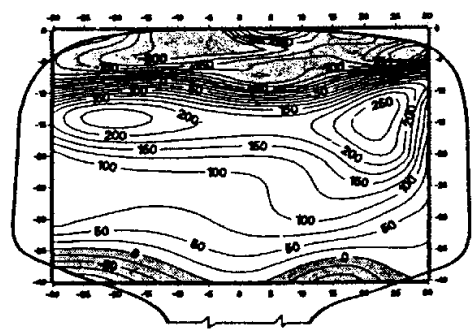

La figure 24 montre une carte des contraintes à l'intérieur d'un rail de chemin de fer usagé [12]. Elle est négative (compression) dans le haut du rail, positive (tension) dans le milieu, et redevient négative dans le bas.

\section{1. 5. Dimension et forme des particules (DNPA)}

L'étude des objets de grandes dimensions (grandes devant les dimensions interatomiques) se fait par diffusion de neutrons à petits angles (DNPA). En fait, il s'agit de diffusion à petits vecteurs $\overrightarrow{\mathrm{Q}}$, c'est à dire de diffusion à petits angles et à grandes longueurs d'onde.

Lorsqu'on scrute ces objets à une échelle qui est grande devant les dimensions de ces objets (petits vecteurs $\vec{Q}$ ), les neutrons ne voient de ces objets qu'un ensemble global, caractérisé par son rayon de giration $R_{G}$. On est alors dans le domaine de Guinier et le facteur de structure correspond au développement suivant:

$$
S(Q)=1-\frac{Q^{2} R_{G}^{2}}{3}+\ldots
$$

C'est pour des vecteurs $\vec{Q}$ qui vont au delà du domaine de Guinier que la fonction $S(Q)$ va devenir spécifique et représenter la forme exacte des particules. 
Pour des particules anisotropes et orientées dans l'échantillon, l'analyse de l'anisotropie de la diffusion, dans les domaines de Guinier, permet de définir différentes longueurs caractéristiques et ainsi remonter à la forme des particules. C'est le cas des bâtonnets de cristal liquide nématique orientés par un champ magnétique et dont les figures de diffusion sont représentés sur la figure 25 [13].

Figure 25: Diffusion par un polymère en bâtonnets alignés par un champ magnétique
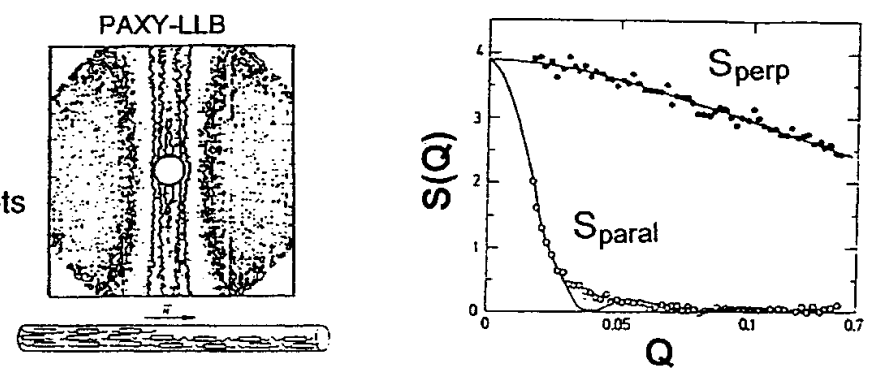

\section{1. 6. Liquides et amorphes}

Dans les liquides comme dans les amorphes il n'y a pas d'ordre à grande distance Le facteur de structure ne présente donc pas de raies de Bragg, mais des anneaux de diffusion, représentatifs de l'ordre à courte distance qui existe entre les atomes. Un exemple en est donné sur la figure 26 pour l'argon liquide [14]. La figure montre aussi la fonction de paires $G(r)$, obtenue à partir de la courbe précédente par transformée de Fourier. Les deux courbes ont la même allure mais pas du tout la même signification car elles correspondent à deux espaces différents.

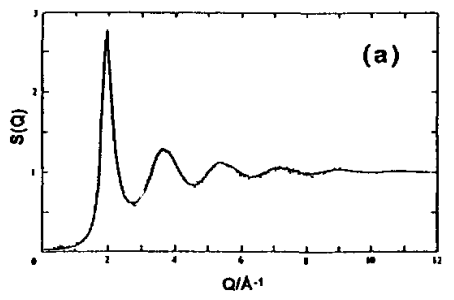

Figure 26: Argon liquide

(a) facteur de structure

(b) fonction de paires

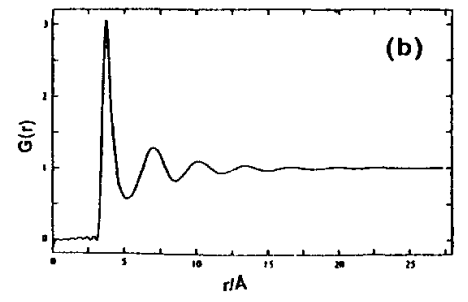

Les courbes de diffusion pour les composés amorphes et pour les liquides se ressemblent beaucoup. Cependant, il est important de réaliser que, contrairement au cas des amorphes, la diffusion par les liquides n'est jamais vraiment élastique. En effet, la section efficace différentielle strictement élastique est donnée par: 


$$
\left(\frac{\mathrm{d} \sigma}{\mathrm{d} \Omega}\right)_{\text {coh }}^{\mathrm{el}}=\sum_{\mathrm{j} \mathrm{j}^{\prime}}\left\langle\overline{\mathrm{b}}_{\mathrm{j}} \overline{\mathrm{b}}_{\mathrm{j}^{\prime}} \mathrm{e}^{-\mathrm{i} \overline{\mathrm{Q}} \cdot \overline{\mathrm{R}}_{\mathrm{j}}(t=0)} \mathrm{e}^{\mathrm{i} \overrightarrow{\mathrm{Q}} \cdot \overline{\mathrm{R}}_{f}(t=\infty)}\right\rangle
$$

et, pour les liquides, il n'y a pas de corrélation entre $\vec{R}_{j}(t=0)$ et $\vec{R}_{j^{\prime}}(t=\infty)$. La courbe de diffusion est donc inélastique, et sa largeur en $\omega$ est d'autant plus grande que $Q$ est plus élevé. On étudie néanmoins la structure des liquides. Ces études sont délicates: on essaye d'intégrer les neutrons diffusés de toutes les énergies pour atteindre la corrélation structurale à $\mathrm{t}=0$, et pour cela il faut appliquer une correction d'inélasticité dite correction de Placzek.

\section{III-2 Voir la dynamique}

Pour étudier les dynamiques, la première approche est de regarder les corrélations temporelles qui existent entre un atome au temps 0 et ce même atome au temps $t$. On s'attache alors à la diffusion incohérente inélastique des neutrons, et cette démarche, toute naturelle dans le cas de mouvements individuels d'atomes peut également se faire dans le cas de mouvements collectifs, même si, à côté de la diffusion incohérente inélastique, existe une diffusion cohérente inélastique, comme on le verra plus loin.

Ce sont donc les atomes qui ont de grosses sections efficaces incohérentes qui sont les meilleurs candidats pour l'étude de la dynamique. Au premier rang desquels on y trouve l'hydrogène, cette fois sous sa forme hydrogène et non plus deutérium.

Les instruments utilisés pour ces études sont essentiellement les spectromètres à temps de vol et, pour les hautes résolutions et les petits transferts d'énergie (mouvements lents), le spectromètre à rétrodiffusion et le spectromètre à spin echo.

Figure 27: Diffusion quasiélastique et diffusion inélastique, d'après [15]

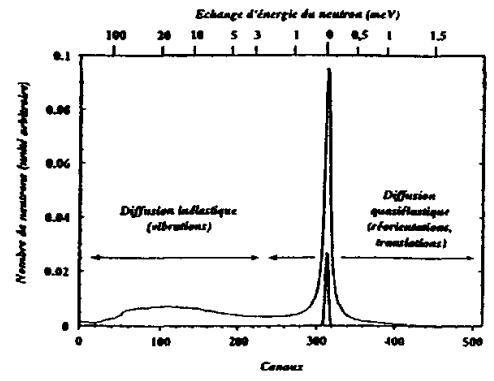

Comme le montre la figure 27, on parle de "diffusion quasiélastique" si le spectre mesuré présente un élargissement du pic élastique et de "diffusion inélastique" si la diffusion est bien séparée de ce pic central élastique.

Nous allons passer en revue les mouvements de translation, de rotation, de vibration et la spectroscopie magnétique.

\section{2. 1. Les mouvements de translation}

Les mouvements de translation se traduisent par une diffusion quasiélastique caractéristique du mouvement. Dans un liquide par exemple, l'élargissement en énergie de $S_{\mathrm{inc}}(\overrightarrow{\mathrm{Q}}, \omega)$ est d'autant plus grand que le vecteur $\mathrm{Q}$ est grand. En effet, à 
petits $Q$, c'est à dire lorsque les neutrons regardent le mouvement d'un atome à grande échelle, pour de grands déplacements, ils voient des mouvements lents résultants des nombreux chocs que subit cet atome: l'élargissement en $\omega$ est faible. Par contre, à grands $Q$, lorsque les neutrons regardent le mouvement à petite échelle, pour de petits déplacements, ils voient un atome presque libre de ses mouvements, en tout cas jusqu'à ce qu'il rencontre ses premiers voisins: le mouvement est rapide et l'élargissement en $\omega$ est important. C'est ce que montre la figure 28 pour l'argon liquide [16].

Figure 28: Dépendance en énergie de l'intensité quasiélastique de l'argon liquide pour différents vecteurs $Q$

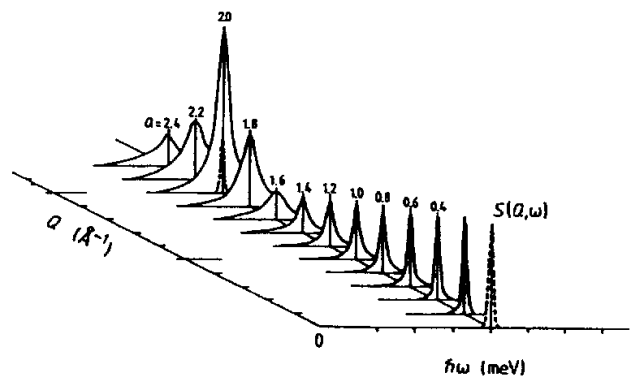

Dans les polymères, les mouvements des chaînons sont beaucoup plus lents. Ils sont observés par des expériences sur un spectromètre à spin echo. C'est alors la fonction intermédiaire $I_{S}(\vec{Q}, t)$ qui est mesurée et sa décroissance en $t$ représente directement la dynamique étudiée. C'est ce qu'illustre la figure 29. Pour un polymère en solution (a), la fonction $I_{S}(\bar{Q}, t)$ tombe à zero selon une exponentielle étirée, plus lentement que les mouvements de diffusion habituels des atomes [17]. Par contre, pour un polymère fondu (b), cette fonction commence par décroitre, puis présente un plateau, reflétant ainsi la très lente corrélation d'un chaînon avec lui même, dans son mouvement de reptation, contraint qu'il est à l'intérieur d'un tube formé par les points d'enchevêtrement des chaînes voisines [18].

Figure 29: Fonction intermédiaire mesurée par écho de spin

(a) pour un polymère en solution

(b) pour un polymère fondu (a)
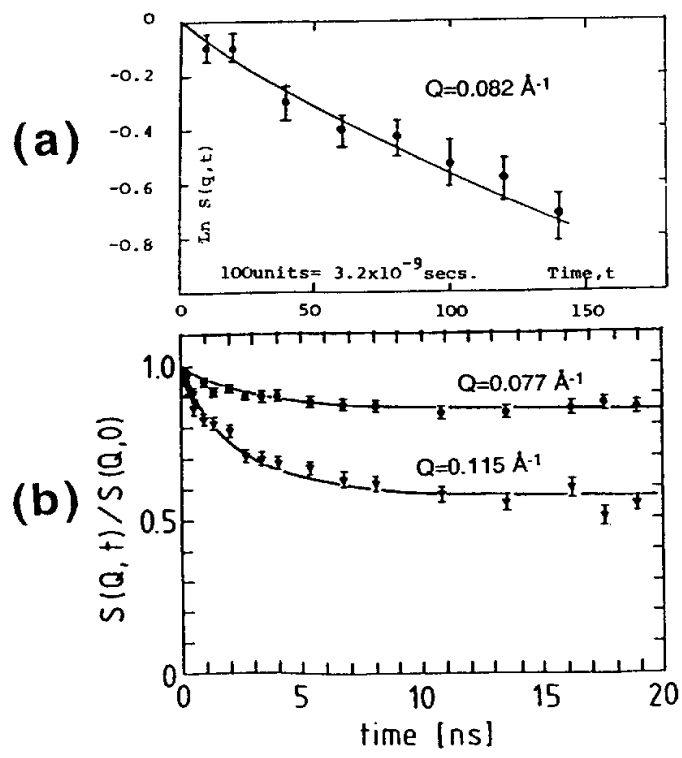


\section{2. 2. Les mouvements de rotation}

Selon les cas, les mouvements de rotation peuvent donner lieu soit à une diffusion inélastique, avec des niveaux bien définis, correspondant à des sauts d'une position à une autre position, soit à une diffusion quasiélastique qui représente une rotation continue. La figure 30 illustre, pour l'ion ammonium de $\mathrm{NH}_{4} \mathrm{ClO}_{4}$, le passage du régime de sauts par effet tunnel à basse température au régime où ces atomes tournent paratiquement librement lorsque la température s'élève [19].

Figure 30; Rotation des ions $\mathrm{NH}_{4}+$ dans $\mathrm{NH}_{4} \mathrm{ClO}_{4}$

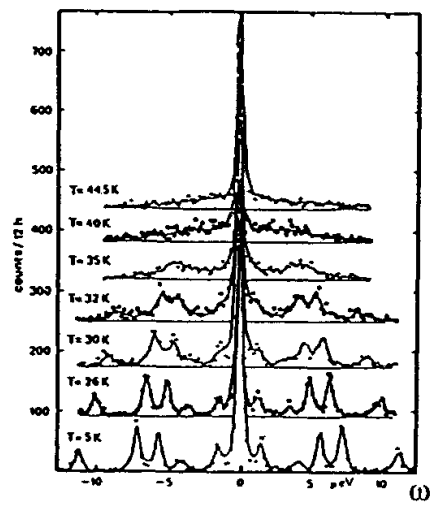

\section{2. 3. Mouvements de vibration et densité de phonons}

Les vibrations d'atomes dans la molécule représentent des énergies très élevées, de l'ordre de quelques centaines de meV. Elles sont rares les sources de neutrons (ISIS, par exemple) qui permettent d'atteindre de tels transferts d'énergie.

Par contre, les phonons dans les solides, comme ce sont des vibrations collectives où les atomes se déplacent ensemble, impliquent des énergies beaucoup plus basses: quelques meV. Bien que ces mouvements soient coordonnés, il est possible de ne suivre le mouvement que d'un atome particulier. La mesure de sa section efficace différentielle incohérente représente alors la densité de phonons $g(\omega)$. C'est un tel exemple que montre la figure 31 pour le vanadium [20].

Figure 31: Densité d'états de phonons du vanadium

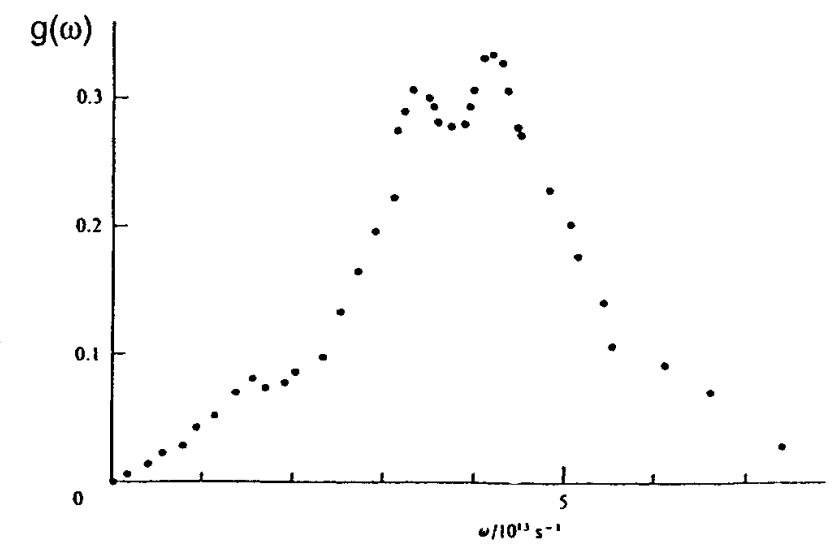




\section{2. 4. Niveaux de champ cristallin et spectroscopie magnétique}

Pour les composés paramagnétiques, l'interaction entre le neutron et le moment magnétique, moment magnétique qui a dans l'échantillon toutes les directions possibles, est par essence incohérente. Comme pour la diffusion incohérente nucléaire, cette diffusion représente la corrélation d'un moment (au temps zero) avec lui-même (au temps t). Sa dépendance en énergie permet d'explorer les transitions de niveau provoquées par l'interaction avec le neutron et représente ainsi une véritable spectroscopie magnétique.

Figure 32: Niveaux de champ cristallin du $\mathrm{Nd}^{3+}$ dans l'oxyde $\mathrm{Nd}_{1-x} \mathrm{Ce}_{x} \mathrm{LaCuO}_{4}$

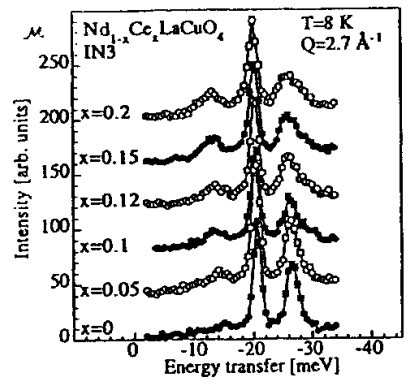

Deux exemples d'une telle spectroscopie sont représentés ici. Dans le premier cas (figure 32), les moments des atomes de terre rare sont trop éloignés pour interagir entre eux et on a accès aux niveaux de champ cristallin [21]. Dans le deuxième cas (figure 33), les moments des 4 atomes de chrome de la molécule de rhodosochlorure de chrome sont fortement couplés entre eux [22]. Le neutron fait alors transiter l'ensemble des 4 moments et la spectroscopie neutronique est alors une spectroscopie moléculaire.

Figure 33: Spectroscopie magnétique moléculaire dans $\mathrm{Cr}(\mathrm{OH})_{6}\left(\mathrm{NH}_{3}\right)_{12} \mathrm{Cl}_{6} .4 \mathrm{H}_{2} \mathrm{O}$

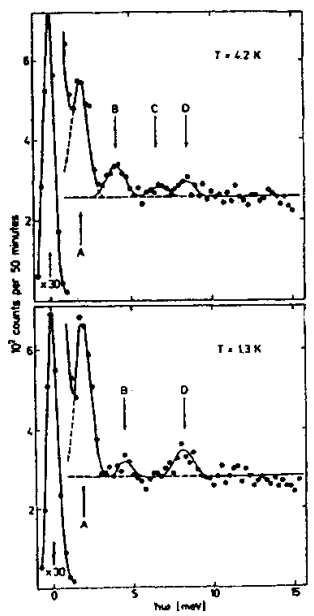

\section{III-3 Voir le couplage entre dynamique et structure}

Du point de vue conceptuel, mais également en pratique, c'est là une des grandes originalités de l'outil neutronique. La section efficace est une fonction à la fois de $\vec{Q}$ et de $\omega$. Le neutron voit le mouvement des atomes à la fois dans l'espace et dans le temps. Il permet d'étudier les couplages entre dynamique et structure. 
Dans un premier temps, on examinera les sections efficaces inélastiques cohérentes qui représentent bien les excitations collectives. Ensuite on se penchera sur les enseignements que l'on peut tirer des sections efficaces incohérentes.

\section{3. 1. Excitations collectives}

Ces excitations collectives recouvrent essentiellement les phonons et les magnons, pour lesquels on mesurera la section efficace inélastique cohérente $\left(\frac{d^{2} \sigma}{d \Omega d E^{\prime}}\right)_{c o h}^{\text {inel }}$ à l'aide d'un spectromètre à temps de vol ou d'un spectromètre triple axe.

1er cas: étude des phonons

Les phonons sont des mouvements coordonnés d'atomes sur une chaîne ou sur un réseau cristallin. Ces mouvements correspondent à des ondes de déplacements, ondes caractérisées par des vecteurs de propagation $\overrightarrow{\mathrm{k}}$.

Les courbes de dispersion $E(k)$ ou $\omega(k)$ sont représentatives des forces de liaisons entre atomes. Elles comportent des branches acoustiques partant de l'origine: la branche longitudinale (LA) correspond à des déplacements parallèles à $\vec{k}$; les deux branches transverses (TA) correspondent, elles, à des déplacements perpendiculaires à $\overrightarrow{\mathrm{k}}$.

Si le cristal comprend plus d'un atome par maille, il existe, en plus des modes acoustiques, des modes optiques (LO et TO), pour lesquels les différents atomes de la maille ne se déplacent pas en phase. Ces modes ont, bien logiquement, une énergie plus élevée que les modes acoustiques.

Figure 34: Courbes de dispersion des phonons dans $\mathrm{CsCl}$

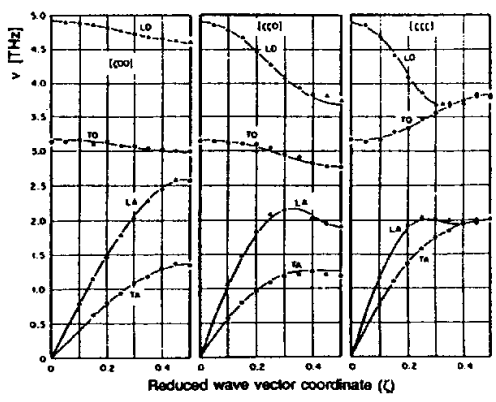

La figure 34 montre les courbes de dispersion mesurées pour le composé $\mathrm{CsCl}$ [23].

Dans le cas des liquides, on ne peut pas parler stricto sensu de phonons. Cependant, les mouvements d'atomes dans les liquides correspondent à des excitations qui ressemblent beaucoup aux branches acoustiques des phonons dans les cristaux. La courbe de dispersion de l'helium liquide $\left({ }^{4} \mathrm{He}\right)$ est représentée sur la figure 35a. Elle présente la particularité d'avoir un minimum, dit minimum de rotons, pour une valeur de $k$ finie. La figure $35 \mathrm{~b}$ montre, pour cette valeur de $k$, la largeur en énergie de cette excitation. Cette largeur, qui augmente lorsque la température croît, indique que la durée de vie de cette excitation particulière est de moins en moins longue lorsque la température s'élève [24]. 
(a)

Figure 35: Helium liquide (a) courbe de dispersion (b) largeur de l'excitation du roton

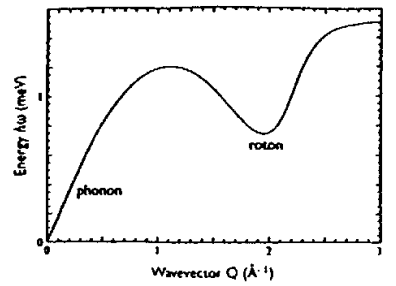

$2^{e m e}$ cas: étude des magnons

A l'instar des phonons, les magnons representent les excitations d'une structure magnétique ordonnée. L'écart à l'ordre magnétique parfait se fait par des ondes de perturbation qui se propagent selon un vecteur d'onde $\overrightarrow{\mathrm{k}}$.

Figure 36: Courbes de dispersion des magnons dans le gadolinium

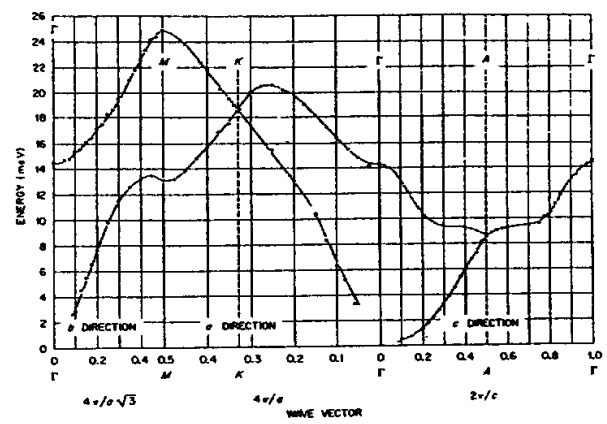

De la même façon que, pour les phonons, les courbes de dispersion $E(k)$ représentaient les forces de liaison entre atomes, pour les magnons, les courbes de dispersion représentent les interactions d'échange entre moments magnétiques. La figure 36 représente les courbes de dispersion de magnons mesurées sur un monocristal de gadolinium, en fait sur un monocristal d'isotope ${ }^{160} \mathrm{Gd}$, car le gadolinium naturel est très absorbant [25].

\section{3. 2. Mouvements Individuels d'atomes}

On suivra dans cette approche la dépendance en $Q$ de la section efficace incohérente. Or cette section efficace, voir formule (95), se compose d'une partie élastique et d'une partie inélastique, et chacune de ces parties peut apporter des informations sur le mouvement.

\section{A travers la diffusion inélastique}

On s'intéressera ici à la variation, en fonction de $Q$, de la largeur inélastique (en $\omega$ ) du pic de diffusion. Pour les problèmes de diffusion, on comparera avec les résultats d'un modèle simple, le modèle de Fick, à un seul paramètre, où le flux d'atomes $\overrightarrow{\mathbf{J}}$ est proportionnel au gradient de la concentration $c$ :

$$
\overrightarrow{\mathrm{J}}=-\mathrm{D} \overrightarrow{\mathrm{grad}} \mathrm{c}
$$


ce qui donne une exponentielle pour la fonction intermédiaire self:

$$
I_{S}(Q, t)=e^{-D Q^{2} t}
$$

et une lorentzienne en $\omega$ pour le facteur de structure dynamique incohérent:

$$
S_{\mathrm{inc}}(\mathrm{Q}, \omega)=\frac{1}{\pi} \frac{\mathrm{DQ}^{2}}{\omega^{2}+\left(\mathrm{DQ}^{2}\right)^{2}}
$$

Figure 37: Largeur en énergie de la diffusion incohérente de l'eau

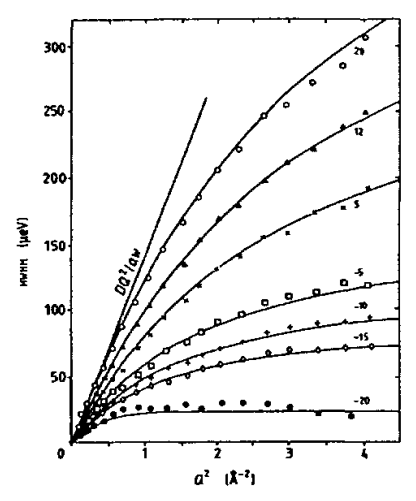

La dynamique de l'eau, liquide et surfondue, a été beaucoup étudiée de cette façon. La figure 37 montre que, d'une façon générale, à petits $Q$, les molécules d'eau suivent la loi de Fick, tandis que à grands $Q$ elles ne la suivent plus [26]. Dans ce cas, il faut faire appel à un modèle plus compliqué, où interviennent deux temps caractéristiques: un temps de saut et un temps de résidence. L'écart à la loi de Fick est d'autant plus fort que la température est plus basse et l'eau plus surfondue.

\section{A travers la diffusion élastique}

Il y a là une situation un peu surprenante où on étudie le mouvement à travers la diffusion élastique. II faut se rappeler que diffusion incohérente élastique ne signifie pas corrélation entre particules immobiles, mais corrélation entre une particule à l'instant 0 et la même particule à $t=\infty$.

$$
\left(\frac{\mathrm{d} \sigma}{\mathrm{d} \Omega}\right)_{\mathrm{inc}}^{\mathrm{el}}=\frac{\sigma_{\mathrm{i}}}{4 \pi} \sum_{\mathrm{j}}\left\langle\mathrm{e}^{-\mathrm{i} \overrightarrow{\mathrm{Q}} \cdot \overrightarrow{\mathrm{R}}_{j}(0)} \mathrm{e}^{\mathrm{i} \mathrm{Q} \cdot \overrightarrow{\mathrm{R}}_{j}(\infty)}\right\rangle
$$

Si l'atome que l'on observe ne s'en va pas à l'infini au cours de son mouvement, (c'est le cas des rotations et des vibrations), la contribution incohérente élastique permet d'étudier ce qu'on pourrait appeler la structure du mouvement.

Pour les rotations, la contribution élastique est appelée le facteur de structure élastique incohérent (EISF en abréviation anglaise). La figure 38 a montre pour le sulfolane la séparation de la section efficace incohérente entre partie élastique et partie quasiélastique. La figure $38 \mathrm{~b}$ compare la variation en $Q$ de l'EISF mesuré avec 
les prédictions fournies par différents modèles de rotation de l'hydrogène dans la molécule [27].

(a)

(b)

Figure 38: Diffusion par le sulfolane (a) séparation entre I'EISF et le quasielastique, (b) variation en $Q$ de l'EISF
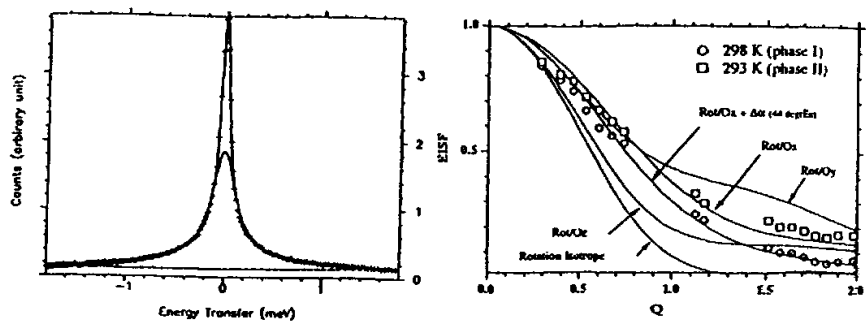

Pour les vibrations, l'effet bien connu est, comme pour le cristal, la présence d'un facteur de Debye-Waller dans l'expression de la section efficace incohérente élastique:

$$
\left(\frac{\mathrm{d} \sigma}{\mathrm{d} \Omega}\right)_{\mathrm{inc}}^{\mathrm{cl}}=\mathrm{N} \frac{\sigma_{\mathrm{inc}}}{4 \pi} \mathrm{e}^{-<\mathrm{u}^{2}>\mathrm{Q}^{2}}
$$

où $\left\langle\mathbf{u}^{2}>\right.$ représente le déplacement quadratique moyen de l'atome qui est à l'origine de la diffusion incohérente. Ce déplacement est en fait une convolution entre le mouvement de vibration propre de l'atome considéré et le mouvement d'ensemble (vibration ou libration) du fragment de molécule comprenant cet atome.

(a)

Figure 39: (a) Diffusion élastique incohérente par une membrane

(b) variations de $\left\langle u^{2}\right\rangle$ avec la température

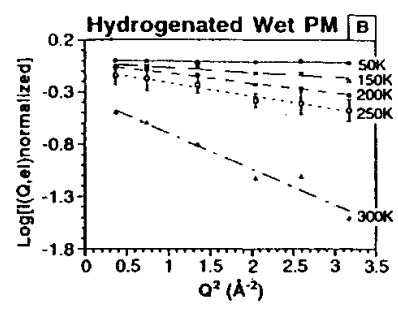

(b)

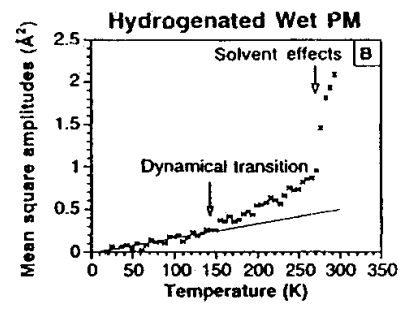

La figure 39a montre, pour différentes températures, la dépendance en $Q$ de la section efficace incohérente élastique diffusée par les atomes d'hydrogène d'une membrane [28] et la figure 39b représente les variations de $\left\langle u^{2}\right\rangle$ en fonction de la température. L'évolution, linéaire à basse température, présente deux transitions : à $150 \mathrm{~K}$ d'abord, transition attribuée à un changement de fonction biologique de la membrane, suivie d'une autre transition à $270 \mathrm{~K}$, attribuée au solvant.

\section{En guise de conclusion: ce qui n'a pas été traité dans cette introduction}

Au cours de cette introduction où a été esquissé un panorama général de l'utilisation des neutrons pour l'étude des matériaux, l'aspect polarisation des neutrons n'a pas du tout été pris en compte. En fait, au lieu d'utiliser l'expression (20) pour la section efficace différentielle, il aurait fallu utiliser l'expression qui se trouve dans la note ${ }^{4}$, 
expression prenant en compte la variable de spin $\sigma$. De ce fait, les formules données dans cette introduction sont essentiellement valables pour des neutrons non polarisés.

Avec des neutrons polarisés, et avec éventuellement une analyse de la polarisation du faisceau diffusé, on peut avoir accès aux composantes $M_{\perp x}, M_{\perp y}, M_{\perp z}$ des moments magnétiques, aux composantes $\mathrm{I}_{x}, \mathrm{l}_{\mathrm{y}}, \mathrm{l}_{\mathrm{z}}$ des spins nucléaires, on peut séparer la diffusion magnétique de la diffusion nucléaire, la diffusion incohérente isotopique de la diffusion incohérente de spin et enfin, dans certains cas, mesurer les composantes magnétiques avec une précision exceptionnelle.

\section{Bibliographie}

[1] "Neutron Cross Sections", BNL-325, édité par D.J. Hughes et J.A. Harvey, USAEC, McGraw-Hill Book, 1955

"Neutron Cross Sections", volume 2, édité par V. McLane, C.L. Dunford et P.F. Rose, Academic Press, 1988

[2] L. Koester et W.B. Yelon, Neutron Diffraction Newsletter, édité par G.H. Lander et M.H. Mueller, 1982

[3] "Theory of Thermal Neutron Scattering" par W. Marshall et S.W. Lovesey, Oxford, Clarendon Press (1971)

[4] "Neutron Cross Sections", volume 1 par S.F. Mughabghab, M. Divadeenam et N.E. Holden, Academic Press (1981)

[5] R. Scherm et B. Fåk, in "Neutron and Synchrotron for Condensed Matter Studies (H.E.R.C.U.L.E.S)", edité par J. Baruchel, J.L. Hodeau, M.S. Lehmann, J.R. Regnard et C. Schlenker, Volume 1, Springer-Verlag, Berlin-Heidelberg et Les Editions de Physique, Les Ulis, 1993, pp 113-143

[6] E.O. Wollan, W.L. Davidson et C.G. Shull, Phys. Rev. 75 (1949) 1348

[7] S.W. Peterson et H.A. Levy, Acta Cryst. 10 (1957) 70

[8] W.F. Kuhs et M.S. Lehmann, J. Phys. Chem. 87 (1983) 4312

[9] C.G. Shull et J. S. Smart, Phys. Rev. 76 (1949) 1256

[10] A. Herpin, P. Meriel et J. Villain, C. R. Acad. Sci. 249 (1959) 1334

[11] C.G. Shull et Y. Yamada, J. Phys. Soc. Japan, 17 BIII (1962) 1

[12] P.J. Webster, K.S. Low, G. Mills et G.A. Webster in "Proc. Mat. Res. Soc. Symp." 166 (1990) 311

[13] M.H. Li, A. Brûlet, J.P. Cotton, P. Davidson, C. Strazielle et P. Keller, J. Phys. II, 4 (1994) 1843

[14] J.L. Yarnell, M.J. Katz, R.G. Wenzel et S.H. Koenig, Phys. Rev. A7 (1973) 2130

[15] M. Bée in "Diffusion Quasiélastique des Neutrons", Ecole Thématique de la Société Française de Neutronique, édité par M. Bée, 2000 , p.1

[16] M. Bée in "Quasielastic Neutron Scattering", Adam Hilger, Bristol and Philadelphia, 1988

[17] L.K. Nicholson, J.S. Higgins et J.B. Hayter in "Neutron Spin Echo, Proceedings, Grenoble 1979", p75, Edité par F. Mezei, Springre-Verlag, Berlin, Heidelberg, New York, 1980

[18] D. Richter, L. Wilner, A. Zirkel, B. Farago, L.J. Fetters et J.S. Huang, Phys. Rev. Let. 71 (1993) 4158

[19] M. Prager, B. Alefeld, A. Heidemann in "Magnetic Resonance and Related Phenomena", édité par H.B. Brunner et al, Groupement Ampère, Heidelberg 1976 [20] W. Glaser, F. Carvalho et G. Ehret in "Inelastic Scattering of Neutrons", IAEA, Vienne, Vol I, p.99, 1965 
[21] M. Gutman, P. Allenspach, F. Fauth, A. Furrer, M. Zolliker, S. Rosenkranz et R.S. Eccleston, Physica B 234-236 (1997) 812

[22] A. Furrer in "Neutron Inelastic Scattering from Isolated Clusters of Magnetic lons", p63, Würenlingen, 1988

[23] H. Bilz et W. Kress in "Phonon Dispersion Relations in Insulators" Springer Series in Solid State Sciences, Vol 10, 1979

[24] K.H. Andersen, J. Bossy, J.C. Cook, O.G. Randl et J.L. Ragazzoni, Phys. Rev. Lett. 77 (1996) 4043

[25] W.C. Koehler, H.R. Child, R.M. Nicklow, H.G. Smith, R.M. Moon et J.W. Cable, Phys. Rev. Lett. 24 (1970) 16

[26] J. Texeira, M.C. Belissent-Funel, S.H. Chen et A.J. Dianoux, Phys. Rev. A31 (1985) 1913

[27] J. Combet, M. Bée, D. Djurado, G. D'Azenza et G. Commandeur, Physica B233 (1997) 95

[28] M. Ferrand, A.J. Dianoux, W. Petry et G. Zaccaï, Proc. Natl. Acad. Sci. USA 90 (1993) 9668 